\title{
Déplacements du front d'un tunnel renforcé par boulonnage prenant en compte le glissement boulon-terrain : approches analytique, numérique et données in situ
}

Laboratoire Géomatériaux-

Département Génie Civil et Bâtiment

(CNRS URA-1652),

École nationale des travaux publics de l'État,

rue Maurice-Audin.

69518 Vaulx-en-Velin Cedex,

France

\section{DIAS}

URGC Géotechnique, Institut national des sciences appliquées de Lyon, 20, avenue Albert-Einstein, 69621 Villeurbanne Cedex,
Dans cet article est proposé un nouveau modèle analytique sur le comportement du front d'un tunnel renforcé par boulonnage, par l'approche d'homogénéisation des milieux périodiques. Ce nouveau modèle s'appuie sur un modèle existant proposé par (Wong et al. 1996, 1997), mais permet de prendre en compte une valeur finie de I'adhérence d'interface boulon/sol, ce qui correspond plus à la réalité. Le comportement d'interface est simulé par un comportement type rigide-plastique parfait, prenant en compte d'un glissement lors du dépassement d'un seuil d'adhérence. Ceci permet notamment d'arriver à une estimation plus correcte de la traction dans les boulons, ainsi que de leur apport sur la réduction des mouvements du terrain. Malgré la prise en compte d'hypothèses moins restrictives, la résolution reste simple, préservant le caractère analytique de la solution. Ce nouveau modèle analytique a été validé, d'une part, par des calculs numériques 3D réalisés à l'aide du logiciel aux différences finies FLAC-3D, et, d'autre part, en confrontant ses prédictions à des mesures in situ en provenance d'un chantier réel - le tunnel de Tartaiguille faisant partie du projet du TGV-Méditerranée. Ces développements représentent des outils simples et efficaces, très utiles au stade du prédimensionnement d'un projet.

\section{Displacement behaviour of a bolt-rein- forced tunnel face with finite ground-bolt bond strength: analytical and numerical approaches, in situ data}

In this paper is proposed a new analytical model on the behaviour of a tunnel face reinforced by bolts, using the homogenisation approach for periodic media. This new model has been developed based on the previous work of (Wong et al. 1996, 1997), and allows to account for a finite bond strength of the soil-bolt interface, which corresponds better to the reality. The interface behaviour is simulated by an elastic-perfectly plastic constitutive relation : interface sliding intervenes when the shear stress reaches the bond strength. This new feature allows notably a more correct estimate of the bolt tension, as well as their quantitative contribution on the displacement reduction of the ground. Despite the consideration of less restrictive hypotheses, the resolution process remains sufficiently simple, thus preserving the analytical character of the solution. This new analytical model has been validated on one hand by $3 D$ numerical calculations using the finite difference code FLAC-3D, and on the other hand by comparing its predictions to in situ data from a construction project - the Tartaiguille Tunnel which forms part of the Mediterranean TGV project. These developments result in simple and efficient design tools, which are very useful at the preliminary design stage of a project. 


\section{Introduction}

Le développement croissant d'infrastructures souterraines implique parfois le creusement de tunnels (ferroviaires ou routiers) dans des conditions géologiques difficiles (terrain peu résistant) entrainant d'importants mouvements de terrain souvent dommageables pour les bâtiments de surface existants. Dans l'optique de limiter ces mouvements et de rendre possible l'excavation en toute sécurité, l'emploi d'un tunnelier n'est pas toujours approprié (tunnels courts, de section trop grande ou non circulaire); on a alors recours à différentes techniques de présoutènement ou confinement du front du tunnel afin d'améliorer les caractéristiques du massif à excaver (Lunardi, 1997) (Peila et al., 1993). Parmi elles, on s'intéresse à l'emploi du boulonnage en fibre de verre pour renforcer le noyau d'avancement (Lunardi, 1993), technique émergente sur les chantiers français et italiens, offrant des avantages certains, parmi lesquels un coût relativement modeste, l'emploi de matériels légers, une grande flexibilité de par son adaptabilité aux conditions géotechniques locales. Du point de vue du fonctionnement mécanique, la forte résistance longitudinale des boulons améliore effectivement les caractéristiques mécaniques du massif; la relative fragilité transversale assure un terrassement aisé.

En matière de dimensionnement, deux approches existent et se complètent. L' ( approche stabilité », basée sur la théorie du calcul à la rupture, permet d'estimer la marge de sécurité par défaut ou par excès mais ne donne aucune information sur les déplacements et notamment sur le mouvement d'extrusion du front. Quant à l' «approche déformations $»$, les méthodes actuelles de dimensionnement du boulonnage frontal les plus utilisées restent empiriques : le tunnel renforcé est souvent modélisé comme un talus armé infiniment long ayant une surcharge correspondant au poids des terres, introduisant ainsi une grande inexactitude sur la géométrie de l'ouvrage.

Certaines méthodes assimilent l'action de renforcement des boulons, a priori anisotrope, à une augmentation isotrope de la cohésion du massif; d'autres remplacent l'action des boulons par une pression de confinement appliquée au front de taille (Labiousse, 1996). L'une ou l'autre de ces approches nécessite une estimation "forfaitaire » de la traction du boulon, souvent prise comme la traction limite à la rupture du boulon modérée par un facteur de sécurité arbitrairement choisi. Or, on conçoit aisément que la traction du boulon doit résulter d'un équilibre qui dépend intrinsèquement des raideurs et résistances relatives du système composite massif et boulons, ainsi que du comportement d'interface. Pire, la faible rigidité des boulons en fibre de verre fait que leur traction n'est souvent qu'un faible pourcentage de leur traction limite. Ces deux approches qui tendent à surestimer l'action des boulons sont donc foncièrement incorrectes et non sécurisantes.

Les modélisations numériques $3 \mathrm{D}$, prenant en compte individuellement chacun des boulons et un comportement réaliste d'interface boulon/terrain, restent souvent le dernier recours pour aboutir à un dimensionnement raisonnablement fiable. Toutefois, ces modélisations nécessitent des calculs longs et onéreux, pas toujours justifiables au stade de l'avant-projet de construction d'un tunnel.
Dans ce contexte, une modélisation théorique du comportement du front de taille d'un tunnel renforcé par boulonnage a déjà été présentée par (Wong et al., 1996, 1997) sous l'hypothèse de la symétrie sphérique, en s'inspirant des travaux antérieurs de Jassionnesse et al. (1996). Cette représentation a donné lieu au développement de nombreux modèles analytiques qui diffèrent suivant le critère et le comportement du sol utilisés :

- critère de Tresca avec comportement élasto-plastique parfait du sol (Wong et al., 1998) ;

- critère de Mohr-Coulomb avec comportement élastoplastique parfait du sol (Wong \& Doanh 1997) ;

- critère de Tresca avec comportement élasto-plastique radoucissant du sol (Subrin 1997);

- critère de Tresca avec longueur finie des boulons (Wong et al., 1999).

L'ensemble de ces modẻles est basé sur un certain nombre d'hypothèses simplificatrices, dont l'homogénéisation des milieux périodiques, la prise en compte d'une pression fictive décroissante simulant l'avancement du front et l'hypothèse d'une adhérence parfaite entre le sol et les boulons. Cette dernière impose que les champs de déplacement des deux matériaux soient les mêmes entraînant inévitablement une surévaluation de la tension dans les boulons. En réalité, il est plus probable que la limite de résistance du scellement soit atteinte avant la plastification des boulons et il existe alors une zone où intervient un glissement à l'interface scellement/terrain (Indraratna et Kaiser, 1990), qui modifierait fondamentalement la répartition de la traction du boulon, comme le montre la figure 1.

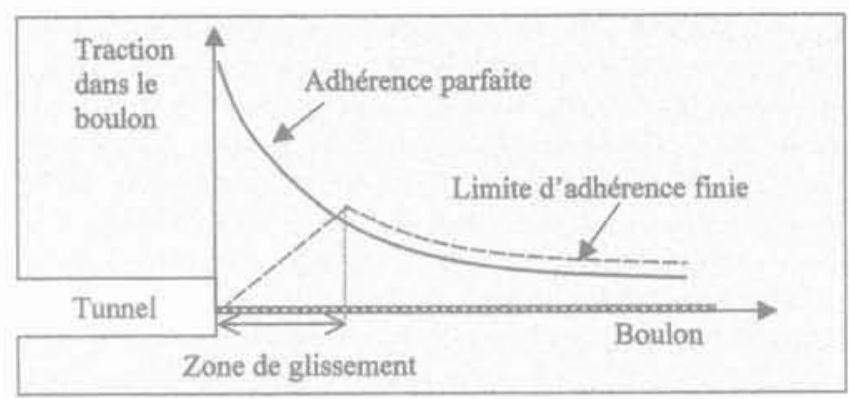

FG.1 Traction le long d'un boulon dans le cas de l'adhérence parfaite et non parfaite.

Bolt tension distribution in the case of finite bond strength.

On propose donc, dans cet article, une nouvelle extension du modèle de base qui prend en compte ce possible glissement à l'interface sol/boulon.

Ce modèle sera validé en comparant ses prédictions à celles issues d'une modélisation numérique 3D par différences finies utilisant le logiciel de calcul FLAC 3D, d'une part, et à des résultats de mesures in situ, d'autre part.

\section{2}

\section{Définition du problème et hypothèses}

Les hypothèses fondamentales sur lesquelles est basé notre modèle sont celles du modèle analytique 
développé par Wong et Jassionnesse (1996) et détaillées ci-après à l'exception de l'adhérence parfaite qui n'est plus vérifiée sur toute la longueur du boulon.

- Le front de taille est assimilé à une surface sphérique où les boulons (de longueur infinie et présents dans le sol dès l'instant initial) sont disposés dans la direction radiale. On suppose que les champs de contrainte, de déformation et de déplacement admettent une symétrie sphérique.

- L'approche d'homogénéisation des milieux périodiques (Greuell, 1993) permet de remplacer le système composite constitué du sol et boulons par un milieu homogène équivalent mais anisotrope en raison de l'action directionnelle du renforcement. On suppose que le tenseur des contraintes macroscopiques peut être décomposé en deux parties, une première due au sol et une deuxième due aux boulons :

$\underline{\underline{\sigma}}=\underline{\underline{\sigma^{s}}}+\underline{\underline{\sigma^{b}}}$

- L'hypothèse principale consiste à supposer que l'histoire de contrainte suivie par un point, tel que le point $A$ (Fig. 2), qui devient le point B quelques instants après le terrassement, peut être identifiée à celle du même point B situé sur le front, considéré fixe et soumis à une pression fictive $\mathrm{P}$ qui décroît de la pression géostatique $\mathrm{P}_{-}=\gamma \mathrm{H}$ à zéro. On introduit le paramètre de chargement $\Delta \mathrm{P}$, monotone croissant de 0 à $\mathrm{P}_{\alpha}$, tel que :

$\Delta P=P_{x}-P_{i}$

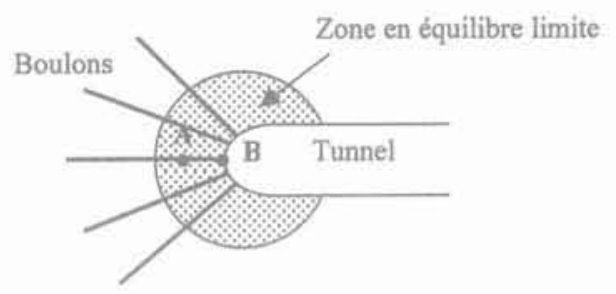

FiG. 2 Représentation schématique de la géométrie du problème simplifié.

Schematic representation of the geometry of the simplified problem.

- On suppose que les comportements du sol et des boulons sont élastiques parfaitement plastiques (le sol obéissant au critère de Tresca). Le massif est supposé élastiquement incompressible $\left(v_{\mathrm{s}}=0,5\right)$. Les relations de comportement seront précisées plus loin.

- On se place dans le cadre d'hypothèses classiques: on suppose le problème quasi statique et on considère le domaine des petites déformations. De plus, en raison de la symétrie sphérique, les tenseurs de contraintes et de déformations sont diagonaux et on a l'égalité parfaite des composantes dans les directions $\theta$ et $\varphi$. Le déplacement est purement radial, ce qui permet de simplifier les relations de compatibilité. En résumé

$\underline{u}=u(r) \underline{e_{r}} \underline{\underline{\sigma}}=\left(\begin{array}{lll}\sigma_{r} & 0 & 0 \\ 0 & \sigma_{\theta} & 0 \\ 0 & 0 & \sigma_{\theta}\end{array}\right) \quad \underline{\varepsilon}=\left(\begin{array}{ccc}\partial_{r} \mathrm{u} & 0 & 0 \\ 0 & \mathrm{u} / \mathrm{r} & 0 \\ 0 & 0 & \mathrm{u} / \mathrm{r}\end{array}\right)$

- Le tunnel est supposé profond $(\mathrm{R} / \mathrm{H}<<1)$ de sorte que le champ de contrainte initial à l'intérieur du terrain est. homogene et isotrope.
L'originalité du modèle dont il est question, par rapport au modèle de base, provient d'une meilleure prise en compte du phénomène d'interface sol/boulon (Spang et Egger, 1990) dont l'hypothèse d'adhérence parfaite précédemment admise est ici remise en question. En effet, on suppose par la suite que la contrainte de cisaillement $\tau$ (Fig. 3) régnant à l'interface boulon/sol (ou plus précisément scellement/sol) est plafonnée à une valeur fixe, appelée limite d'adhérence ou seuil de contrainte de scellement, notée $q_{s}$ (Wong et Larue, 1998). Une fois cette limite atteinte, un glissement se produit alors à l'interface (discontinuité de déplacement) et la contrainte de cisaillement demeure constante égale à $q_{\mathrm{s}}$; autrement dit, elle a un comportement rigide plastique. La prise en compte de ce comportement d'interface conduit à l'apparition d'une zone de décollement entre sol et boulon dès le début du chargement.

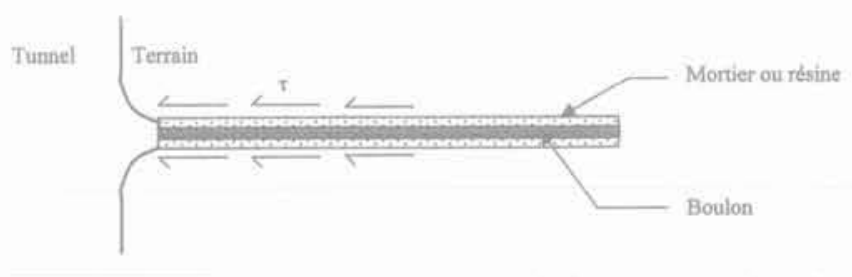

FIG 3 Représentation schématique de la contrainte de cisaillement à l'interface scellement/terrain.

Schematic representation of the shear stress at the grout-ground interface.

Le choix d'un glissement scellement/terrain provient de résultats d'essais d'arrachement in situ, où on constate le plus souvent que les boulons et le mortier restent solidaires, et le glissement intervient à l'interface scellement/sol.

Traduit en termes mathematiques, tant que $\tau<q_{s}$, le contact entre le sol et les boulons est supposé parfait. Ils admettent donc les mêmes déplacements et les mêmes déformations radiaux, soit :

$$
\varepsilon^{b}(r)=\varepsilon_{r}^{3}(r) \text { noté } \varepsilon_{p}(r)
$$

D'où :

$$
T_{b}(r)=\frac{E_{b} S_{b} \varepsilon_{r}(r) \operatorname{si} \varepsilon_{r} \leq \varepsilon_{y b}}{\sigma_{y b} S_{b} \operatorname{si} \varepsilon_{r}>\varepsilon_{y b}}
$$

Dès que $\tau=q_{s^{\prime}}$ le phénomène de décollement apparait. La répartition des efforts dans chaque boulon suit la loi :

$\frac{d T_{b}}{d r}=p q_{s}=R_{a}$

où $\mathrm{p}$ est le périmètre de l'interface scellement-terrain (circonférence du forage) supposé cylindrique et $\mathrm{R}_{\text {la }}$ résistance unitaire à l'arrachement du boulon (en $\mathrm{kN} / \mathrm{ml}$ ).

En supposant qu'il n'y a pas de plaque d'ancrage en paroi (i.e. $T_{b}(R)=0$ ), ce qui est effectivement le cas du boulonnage frontal, on obtient, après intégration, la traction des boulons dans la zone de déchaussement :

$$
T_{b}(r)=\frac{K T_{y b}}{\Omega}\left(\frac{r}{R}-1\right)
$$


où $K=\frac{d_{b} p q_{s} R}{C}$ et $\Omega=d_{b} S_{b} \frac{\sigma_{y b}}{C}$, paramètres adimensionnels, traduisent respectivement la résistance de l'interface et l'apport de résistance (anisotrope) du boulonnage rapportés à la résistance du terrain.

Compte tenu de toutes ces hypothèses, le problème revient à considérer une cavité sphérique sur la paroi de laquelle est appliquée une pression interne $\mathrm{P}$ monotone décroissante de la pression géostatique $\mathrm{P}$ à 0 . Le milieu est infini, homogène et anisotrope. Initialement, les déplacements et déformations sont nuls $(\underline{u}=\underline{0}$ et $\underline{\varepsilon}=\underline{\underline{0}})$ tandis que le champ de contrainte est homogène et isotrope $\left(\underline{\underline{\sigma}}_{x}=-\mathrm{P}_{\infty} \frac{1}{\underline{\underline{x}}}\right)$.

\section{3}

\section{Loi de comportement homogénéisée}

\section{Loi de comportement élastique}

En élasticité, le tenseur de contrainte du sol seul obéit à la loi de Hooke :

$\underline{\underline{\sigma^{s}}}=2 \mu_{s} \cdot \underline{\underline{\varepsilon}}+\lambda_{s} \operatorname{tr}(\underline{\underline{\varepsilon}})+\underline{\underline{\sigma_{x}}}$

La traction d'un boulon $T_{b}(r)$ rapportée à la surface tributaire $\frac{r^{2}}{d_{b} R^{2}}$ (Greuell, 1993) donne le tenseur de contrainte uniaxial dû aux boulons :

$\underline{\underline{\sigma^{b}}}=\sigma_{0}(r) \underline{e_{r}} \otimes \underline{e_{r}} \quad$ avec $\quad \sigma_{0}(r)=d_{b}\left(\frac{R}{r}\right)^{2} T_{b}(r)$

En combinant les relations (1), (7) et (8), on aboutit à la relation suivante liant les composantes des vitesses de contrainte et celles de déformation

$$
\left[\begin{array}{c}
\dot{\sigma}_{r} \\
\dot{\sigma}_{\theta}
\end{array}\right]=\left[\begin{array}{cc}
\lambda_{s}+2 \mu_{s}+E_{s} \beta \frac{R^{2}}{r^{2}} & 2 \lambda_{s} \\
\lambda_{s} & 2\left(\lambda_{s}+\mu_{s}\right)
\end{array}\right]\left[\begin{array}{c}
\dot{\varepsilon}_{r} \\
\dot{\varepsilon}_{\theta}
\end{array}\right]
$$

en posant $\beta=d_{b} S_{b} \frac{E_{b}}{E_{c}}$, paramètre adimensionnel traduisant l'apport de rigidité des boulons.

En tenant compte de $\mathrm{v}_{\mathrm{s}}=0,5$ et en inversant la relation (9), on arrive à :

$$
\left[\begin{array}{c}
\dot{\varepsilon}_{r} \\
\dot{\varepsilon}_{\theta}
\end{array}\right]=\frac{1}{E_{s}}\left(1+\beta \frac{R^{2}}{r^{2}}\right)^{-1}\left[\begin{array}{cc}
1 & -1 \\
-1 / 2 & 1 / 2
\end{array}\right]\left[\begin{array}{l}
\dot{\sigma}_{r} \\
\dot{\sigma}_{\theta}
\end{array}\right]
$$

\section{Comportement plastique}

Le sol est supposé de comportement élastoplastique parfait, obéissant au critère de Tresca :

$$
f\left(\underline{\underline{\sigma^{s}}}\right)=\sigma_{r}^{s}-\sigma_{\theta}^{s}-2 C \leq 0
$$

Le comportement des boulons est également élastoplastique parfait, décrit par la relation (4).
En tenant compte des relations (1), (8) et (11) ci-dessous, le critère de résistance du composite massif + boulons s'écrit suivant les contraintes macroscopiques:

$f(\underline{\underline{\sigma}})=\sigma_{r}-\sigma_{\theta}-2 C-\sigma_{0}(r) \leq 0$

\section{Loi d'écoulement}

Le tenseur des déformations plastiques est obtenu en supposant que la règle d'écoulement est associative, ce qui implique : $\operatorname{tr}\left(\underline{\underline{\dot{\varepsilon}^{p}}}\right)=0$ pour le critère de Tresca adopté. De plus, en raison de la symétrie sphérique, les déviateurs $\sigma_{\tau}-\sigma_{\theta}$ et $\sigma_{\tau}-\sigma_{\theta}$ atteignent simultanément le critère, on est donc en régime d'arête, ce qui implique :

$\underline{\underline{\varepsilon^{p}}}=\frac{1}{2} \zeta\left(\partial_{g} f_{r \theta}(\underline{\underline{\sigma}})+\partial_{\underline{g}} f_{r \phi}(\underline{\underline{\sigma}})\right)$ avec $f_{u}(\underline{\underline{\sigma}})=\sigma_{i}-\sigma_{j}-2 C-\sigma_{0}(r)$

soit sous forme matricielle :

$\underline{\underline{\dot{\varepsilon}^{p}}}=\dot{\zeta}\left(\begin{array}{ccc}1 & 0 & 0 \\ 0 & -1 / 2 & 0 \\ 0 & 0 & -1 / 2\end{array}\right)$

\section{4}

\section{Équations fondamentales du problème}

\section{Équation d'équilibre}

L'équation d'équilibre exprimée suivant la contrainte totale $\underline{\sigma}=\underline{\sigma^{f}}+\underline{\sigma}^{b}$ est :

$r \hat{\theta}_{r} \sigma_{r}+2\left(\sigma_{r}-\sigma_{\theta}\right)=0$

Dans la zone avec déchaussement, l'équation d'équilibre du point de vue du sol seul, est:

$$
r \partial_{r} \sigma_{r}^{5}+2\left(\sigma_{r}^{s}-\sigma_{\theta}^{s}\right)+d_{b} p q_{s} \frac{R^{2}}{r}=0
$$

où $d_{b} p q_{s} \frac{R^{2}}{r}$ est une force volumique extérieure au sol correspondant à la moyenne sur la cellule de base de la contrainte de cisaillement d'interface exercée par les boulons sur le sol (Stille, 1989).

\section{Conditions initiales}

A l'instant initial $(t=0)$, lorsque $\mathrm{P}_{1}=\mathrm{P}_{\mathrm{N}^{\prime}}$ on $\mathrm{a}$, en tout rayon :

$$
\begin{aligned}
& \underline{\underline{\sigma}}=-P_{\infty} \frac{1}{=} \\
& \underline{\underline{E}}=\underline{\underline{0}} \\
& \underline{\underline{u}}=\underline{0}
\end{aligned}
$$

\section{Conditions aux limites}

A tout instant $t>0\left(\mathrm{P}_{-}<\mathrm{P}_{1}<0\right)$, les contraintes radiales vérifient

$\sigma_{r}(\infty, t)=-P_{\infty}$

$\sigma_{r}(R, t)=-P_{i}$ 
En intégrant l'équation $\underline{\underline{\varepsilon}}=\underline{\underline{\dot{\varepsilon}}}+\underline{\underline{\dot{\varepsilon}^{p}}}$ entre l'instant initial $\mathrm{t}=0$ et un instant ultérieur $\mathrm{t}>0$ et, compte tenu des relations (10) et (14), on obtient les équations suivantes:

$$
\begin{aligned}
& \frac{\partial u}{\partial r}=\varepsilon_{r}=\frac{1}{E_{s}}=\left(1+\beta \frac{R^{2}}{r^{2}}\right)^{-1}\left(\sigma_{r}-\sigma_{\theta}\right)+\zeta \\
& \frac{u}{r}=\varepsilon_{\theta}=\varepsilon_{\varphi}=\frac{1}{2 E_{s}}\left(1+\beta \frac{R^{2}}{r^{2}}\right)^{-1}\left(\sigma_{\theta}-\sigma_{r}\right)-\frac{\zeta}{2}
\end{aligned}
$$

En combinant ces deux équations, on vérifie bien que la trace du tenseur de déformation soit nulle c'està-dire $\operatorname{tr}(\underline{\underline{\varepsilon}})=\operatorname{tr}\left(\underline{\underline{\varepsilon^{e}}}\right)+\operatorname{tr}\left(\underline{\underline{\underline{\varepsilon^{p}}}}\right)=0$, la nullité du premier terme venant de $v_{s}=0,5$ et celle du second est une conséquence de la loi d'écoulement adoptée. Il vient donc:

$$
\frac{\partial u}{\partial r}+2 \frac{u}{r}=0
$$

en tout point du massif et à chaque instant, d'où :

$$
u=-\frac{B R^{3}}{r^{2}} \quad \text { ou encore } \varepsilon_{r}=\frac{2 B R^{3}}{r^{3}}
$$

où $\mathrm{B}$ est une constante d'intégration adimensionnelle indépendante de $r$.

\section{5}

\section{Évolution des zones élastoplastiques (cas de référence)}

Une étude complète a permis de mettre en évidence que trois scénarios (appelés cas A, B et C), correspondant chacun à une séquence d'apparition des zones élastoplastiques, sont envisageables pour décrire le comportement du massif composite entre l'instant $t=0$ où le chargement $\Delta \mathrm{P}$ vaut 0 et l'instant final où le chargement vaut $\mathrm{P}_{\mathrm{m}}$. Ces trois scénarios diffèrent suivant les valeurs des paramètres adimensionnels $\beta, \Omega$ et K considérées.

Une étude paramétrique effectuée en prenant des plages de valeurs réalistes pour $\beta, \Omega$ et $K$ montre que le scénario A est le plus probable en pratique. On choisit donc, par la suite, de ne détailler les développements théoriques que pour ce cas A, pris comme le cas de

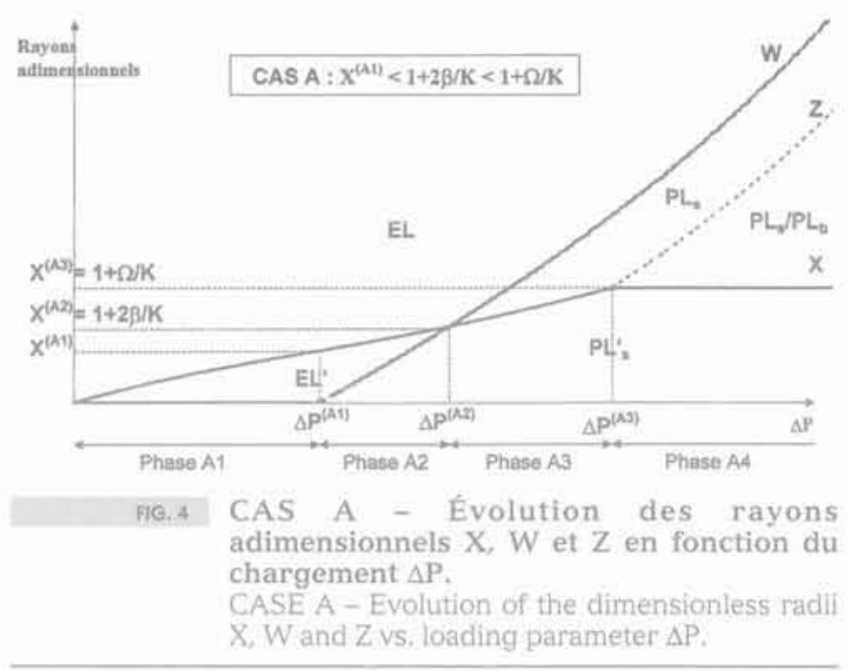

référence, dont les différentes séquences d'événements correspondantes sont présentées dans le tableau 1.

Les cas B et $\mathrm{C}$ seront résumés plus loin.

Dans ce cas de référence, dont les conditions d'application seront explicitées plus loin, une première zone EL', dans laquelle il y a déchaussement à l'interface sol/boulon, apparait dès le début du chargement en paroi et se développe sur un rayon égal à x. Dans cette première phase, les deux composants restent élastiques. A un seuil de chargement $\Delta \mathrm{P}^{\text {(Ai) }}$, une deuxième zone se développe à partir de la paroi (zone $\mathrm{PL}_{\mathrm{s}}$ ), sur une distance égale à $\mathrm{W}$, où le sol est plastifié. Après un seuil critique $\Delta \mathrm{P}^{(\mathrm{A} 2)}$, w dépasse $\mathrm{x}$, ce qui fait apparaitre une nouvelle zone plastique $\mathrm{PL}$ où le sol manifeste un comportement plastique sans qu'il y ait descellement. Au-delà d'un chargement critique $\Delta P^{(A 3)}$, le boulon se plastifie à son tour, donnant naissance à une quatrième et dernière zone, PL/PL, où les deux composants ont un comportement plastique, en adhérant parfaitement l'un à l'autre. Cette dernière phase se poursuit jusqu'au déconfinement total lorsque P s'annule complètement.

La figure 4 exprime d'une manière plus synthétique la séquence d'événements relative au cas A de référence dans le plan $r / \mathrm{R}-\Delta \mathrm{P}$.

Le traitement des cas B et $\mathrm{C}$ suit le même principe ; seuls les résultats principaux sont donc résumés en annexe.

TABLEAUI Évolution des zones plastiques au cours du chargement pour le cas A de référence.

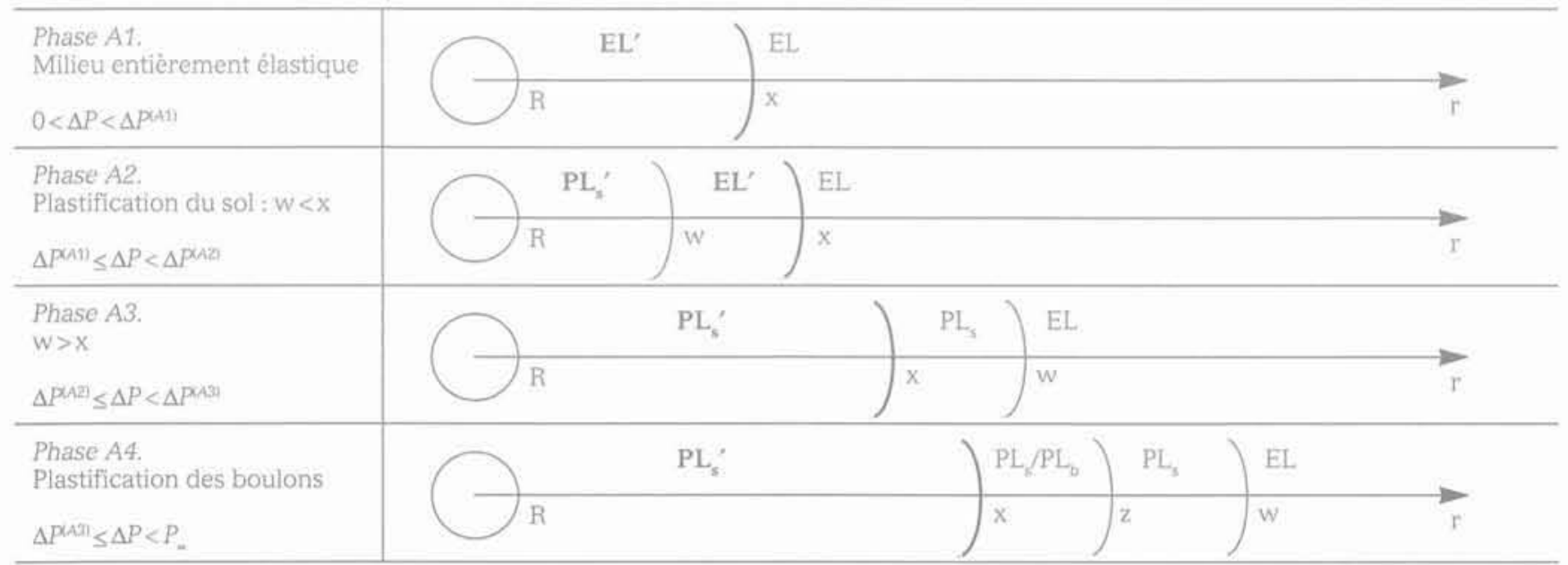


TABLEAUIII Conditions à vérifier pour chacun des trois scénarios envisageables.

\begin{tabular}{l|c|c}
\multirow{2}{*}{ Scénarios possibles } & \multicolumn{2}{|c}{ Conditions } \\
\cline { 2 - 3 } & \multicolumn{1}{|c}{ Phénomènes physiques } & Écriture mathématique équivalente \\
\hline CAS A & $\begin{array}{l}\text { "Le sol plastifie avant les boulons } \\
\text { Les boulons plastifient lorsque } w>x\end{array}$ & $X^{\text {(a11 }}<1+2 \beta / K<1+\Omega / K$ \\
\hline CAS B & $\begin{array}{l}\text { *Le sol plastifie avant les boulons } \\
\text { Les boulons plastifient lorsque } w<X\end{array}$ & $X^{\text {(A11) }<1+\Omega / K<1+2 \beta / K}$ \\
\hline CASC & "Les boulons plastifient avant le sol & $1+\Omega / K<X^{(k 1)<1+2 \beta / K}$ \\
\hline
\end{tabular}

- Voir figures 4 et A1.

*t Voir figure A2.

Les phénomènes physiques qui amènent à considérer trois scénarios possibles sont explicitées dans le tableau II. On y donne également les conditions d'inégalités mathématiques qui régissent chacun d'eux, sachant que celles-ci seront développées par la suite.

\section{6}

\section{Résolution phase par phase du cas A (cas de référence)}

Dans ce paragraphe est présentée la résolution en détail du cas $A\left(X^{(A 1)}<1+2 \beta / K<1+\Omega / K\right)$.

\section{1}

\section{Phase $\mathrm{A} 1$ : deux zones élastiques $\mathrm{EL}^{\prime}(\mathrm{R}, \mathrm{x})$ et $E L(x, \infty)$}

Dans cette phase, le sol et les boulons ont un comportement élastique en tout point.

Dès l'instant initial, une zone de descellement EL' apparait en paroi, où on a rupture de scellement, et se propage à partir du front sur une distance x. Au-delà, les deux matériaux constitutifs adhèrent parfaitement ensemble.

Dans la zone élastique extérieure EL, en substituant (20) dans (17) avec $\zeta=0$ et en éliminant $\sigma_{\theta}$ grâce à l'équation d'équilibre (15), on obtient l'équation différentielle de la seule variable $\sigma_{r}$ :

$$
\partial_{r} \sigma_{r}+\frac{4 B R^{3} E_{s}}{r^{4}}\left(1+\frac{\beta R^{2}}{r^{2}}\right)=0
$$

D'où, d'après la prise en compte des conditions aux limites en $r \rightarrow \infty$;

$$
\sigma_{\tau}=-P_{\infty}+4 B E_{\mathrm{s}}\left(\frac{\beta R^{5}}{5 r^{5}}+\frac{R^{3}}{3 r^{3}}\right)
$$

Pour déterminer la constante $\mathrm{B}$, on écrit la continuité de $T_{b}(r)$ au rayon $x$, c'est-à-dire que $T_{b}(x)=T_{b}\left(x^{+}\right)$ où le membre de gauche provient de l'expression $(6)$ et celui de droite de l'expression (4). On obtient aisément, en fonction du rayon adimensionnel $\mathrm{X}=\mathrm{x} / \mathrm{R}$ :
$B=\frac{K C(\mathrm{X}-1) X^{3}}{2 \beta E_{s}}$

Dans la zone élastique intérieure EL', la relation liant la déformation radiale du sol et les contraintes de ce dernier s'écrit :

$$
\partial_{r} u=\varepsilon_{r}=\frac{1}{E_{s}}\left(\sigma_{r}^{s}-\sigma_{\theta}^{s}\right)
$$

En combinant cette relation avec l'équation d'équilibre (16) du sol et l'expression (20) de $\varepsilon_{r}$, on obtient:

$$
\partial_{r} \sigma_{r}^{s}+\frac{4 E_{s} B R^{3}}{r^{4}}+d_{b} p q_{s} \frac{R^{2}}{r^{2}}=0
$$

D'où, en tenant compte de la condition en paroi $\sigma_{r}^{5}(\mathrm{R})=-\mathrm{Pi}$ :

$$
\sigma_{r}^{5}=-P_{i}+\frac{4 E_{s} B}{3}\left(\frac{R^{3}}{r^{3}}-1\right)+K C\left(\frac{R}{r}-1\right)
$$

En superposant la contrainte homogénéisée $\sigma_{0}(\mathrm{r})$ due aux boulons, et compte tenu des relations (6) et (8), on obtient l'expression de la contrainte homogénéisée:

$$
\sigma_{r}=\sigma_{r}^{s}+\sigma_{0}(r)=-P_{j}+\frac{4}{3} B E_{s}\left(\frac{R^{3}}{r^{3}}-1\right)-K C\left(\frac{R}{r}-1\right)^{2}
$$

Le rayon $x$ se détermine en écrivant la continuité de $\sigma_{\text {r en }} \mathrm{x}$; soit $\sigma_{\tau}\left(\mathrm{x}^{-}\right)=\sigma_{\mathrm{c}}\left(\mathrm{x}^{+}\right)$, où le membre de gauche provient de l'expression (27) de $\sigma$ dans la zone EL' et celui de droite de l'expression (22) de $\sigma$. dans la zone EL. Ce qui s'écrit, en simplifiant par les valeurs adimensionnelles $K, \beta$, X et $\Delta P^{*}=\Delta P / C$ :

$$
\Sigma_{1}(X, \Delta P)=\frac{K C(X-1)}{X^{2}}\left(\frac{2}{3} \frac{X^{5}}{\beta}+X-\frac{3}{5}\right)-\Delta P=0
$$

On démontre facilement qu'on a $\Sigma_{1}(1)=-\Delta \mathrm{P}^{*}<0$, $\Sigma(\infty) \rightarrow \infty$ et $\Sigma_{1}^{\prime}(X>1)>0$, l'équation ci-dessus admet donc une et une racine unique dans l'intervalle $(1, \infty)$. La détermination de $\mathrm{X}$ ne nécessite qu'un simple calcul numérique.

\section{Condition de la fin de la phase A1}

La fin de la phase élastique se produit lorsqu'il y a début de plastification du sol c'est-à-dire lorsque le critère de Tresca est tout juste atteint. Soit : 
$\Delta P$.

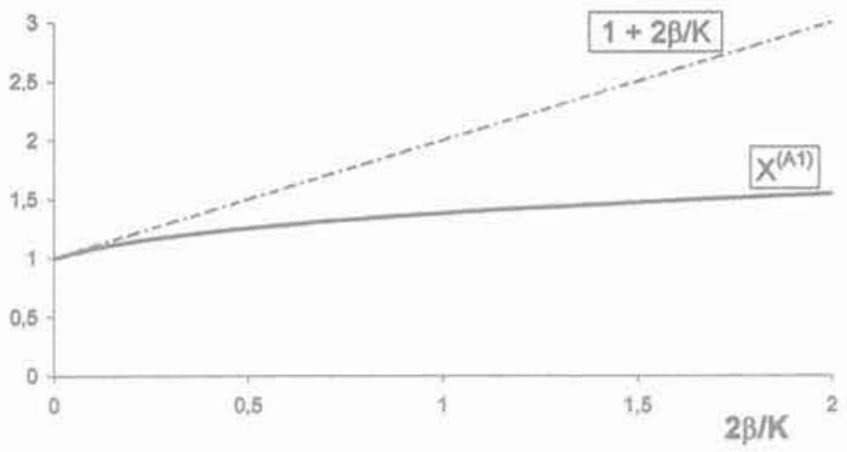

EG.5 Evolution du rayon $X^{(A 1)}$ caractérisant la fin de la phase élastique en fonction de $2 \beta / K$. Evolution of the radius $\mathrm{X}^{(\mathrm{Ar})}$ marking the end of the elastic phase depending on $2 \beta / K$.

$f\left(\underline{\underline{\sigma^{s}}}\right)=\sigma_{r}^{s}-\sigma_{\theta}^{s}-2 C=0$ avec $\sigma_{r}^{s}-\sigma_{\theta}^{s}=E_{s} \varepsilon_{r}^{s}=\frac{2 B R^{3} E_{s}}{r^{3}}$

Ce dernier terme étant monotone décroissant en $\mathrm{r}$, cette condition est d'abord atteinte en paroi pour $\mathrm{r}=\mathrm{R}$, ce qui s'écrit, après avoir substitué la valeur (23) de B et après avoir simplifié :

$X^{4}-X^{3}-\frac{2 \beta}{K}=0$

Cette équation est d'ordre 4 et peut donc être résolue analytiquement selon Abel; elle possède une seule racine réelle positive dépendant de $2 \beta / K$, notée $X^{(A 1)}$, dont l'expression (assez complexe) se détermine par un simple calcul formel. La figure 5 ci-dessus donne l'évolution de la valeur de cette racine en fonction de $2 \beta / K$.

On peut démontrer aisément que $1<X^{(A)}<1+\frac{2 \beta}{K}$ pour $\beta$ et $\Omega$ positifs.

La fin de la phase 1 se produit lorsque le chargement $\Delta \mathrm{P}$ atteint le seuil $\Delta \mathrm{P}^{(\mathrm{A}) \mathrm{l}}$ qui vérifie l'équation $\Sigma_{1}\left(X^{\lceil A 1}, \Delta P^{(A 11}\right)=0$. Soit :

$$
\Delta P^{(A 1)}=\frac{K C\left(X^{(A 1)}-1\right)}{X^{(A 1)^{2}}}\left[\frac{2}{3 \beta} X^{(A 1)^{5}}+X^{(A 1)}-\frac{3}{5}\right]
$$

\section{2}

\section{Phase $\mathrm{A} 2$ : zone plastique $\mathrm{PL}$ ' $(R, w)$, zones élastiques $E L^{\prime}(w, x)$ et $E L(x, \infty)$}

Dans la zone élastique extérieure EL, $\sigma$ admet la même expression (22) qu'en phase A1. La continuité de $T_{b}$ en $x$ donne, de plus, la même expression (23) de B.

Dans la zone plastique $\mathrm{PL}^{\prime}$, en remplaçant le critère de Tresca $\sigma_{r}^{5}-\sigma_{\theta}^{s}-2 C=0$ pour le sol, dans l'équation d'équilibre (16) du sol et en tenant compte de la condition en paroi $\sigma^{\mathrm{s}}(\mathrm{R})=-\mathrm{Pi}$, on obtient après résolution :

$$
\sigma_{r}^{s}=-P_{i}-4 C \operatorname{Ln} \frac{r}{R}+K C\left(\frac{R}{1}-1\right)
$$

D'où la contrainte radiale macroscopique :

$\sigma_{r}=\sigma_{r}^{s}+\sigma_{0}(r)=-P_{i}-4 C \operatorname{Ln} \frac{r}{R}-K C\left(\frac{R}{r}-1\right)^{2}$

Dans la zone élastique EL', l'équation différentielle (25) sur $\sigma_{\mathrm{r}}^{\mathrm{s}}$ est toujours valable. On obtient, après résolution:

$\sigma_{r}^{5}=\frac{4 E_{s} B R^{3}}{3 r^{3}}+\frac{K C R}{r}+D C$

où $\mathrm{D}$ est une constante d'intégration sans dimension.

D'où :

$\sigma_{r}=\sigma_{r}^{s}+K C\left(\frac{r}{R}-1\right)\left(\frac{R}{r}\right)^{2}=\frac{4 E_{s} B R^{3}}{3 r^{3}}+\frac{K C R}{r}\left(2-\frac{R}{r}\right)+D C$

La continuité de $\sigma^{5}$ en $W, \sigma^{5}\left(W^{-}\right)=\sigma^{5}\left(W^{*}\right)$, où le membre de gauche provient de l'expression (32) de $\sigma_{\text {s }}^{5}$ dans la zone PL' 'et celui de droite de l'expression (34) de $\sigma_{r}^{s}$ dans la zone EL', donne, après simplification:

$D+\frac{P_{i}}{C}+4 L n W+K\left[1+\frac{2}{3 \beta}(X-1)\left(\frac{X}{W}\right)^{3}\right]=0$

La continuité de $\sigma_{\text {e }}$ en $\mathrm{x}, \sigma_{\mathrm{f}}\left(\mathrm{x}^{-}\right)=\sigma_{-}\left(\mathrm{x}^{+}\right)$, où le membre de gauche provient de l'expression (35) de $\sigma_{r}$ dans la zone EL' et celui de droite de l'expression (22) de $\sigma_{r}$ dans la zone EL. Ce qui donne, après simplification:

$D=-\frac{P_{x}}{C}+K \frac{3-8 X}{5 X^{2}}$

La détermination des rayons inconnus $w$ et $x$ se déroule comme suit. La première équation vient du fait que le critère de plasticité du sol est tout juste atteint en $w^{+}$. Les contraintes $\sigma_{f}^{5}$ et $\sigma_{0}^{5}$ dans la zone EL obéissant à la loi de comportement élastique linéaire, on a donc, en introduisant le rayon adimensionnel $W=W / R$ :

$\sigma_{r}^{s}-\sigma_{\theta}^{s}=E_{s} \varepsilon_{r}^{s}(w)=E_{s} \frac{2 B}{W^{3}}=2 C$

En remplaçant par la valeur (23) de B, on obtient l'expression de W :

$W^{3}=\frac{1}{2 \beta} K(X-1) X^{3}$

La deuxième équation se déduit en combinant les équations (36) et (37) et en remplaçant W par la valeur ci-dessus ; il en résulte l'équation suivante sur X:

$\Sigma_{2}(X, \Delta P)=\frac{\Delta P}{C}-\frac{4}{3} \operatorname{Ln}\left[\frac{K X^{3}(X-1)}{2 \beta}\right]-K\left(1+\frac{3-8 X}{5 X^{2}}\right)-\frac{4}{3}=0$

De même que précédemment, cette équation possède une et une seule racine qui se détermine facilement par un simple calcul numérique du fait de la décroissance monotone de la fonction. Elle définit une fonction implicitement monotone croissante $X(\Delta P)$.

\section{Condition de la fin de la phase A2}

La fin de la phase A2 a lieu lorsque le rayon w de plasticité du sol rattrape le rayon $\mathrm{x}$ de déchaussement, soit mathématiquement $\mathrm{W}=\mathrm{X}$; d'après l'équation (39), ceci a lieu pour la valeur particulière $X^{(\hat{n} 2)}$, vérifiant:

$X^{(A 2)}=1+\frac{2 \beta}{K}$ 
ce qui correspond, d'après (40), à une valeur de chargement égale à :

$$
\Delta P^{(A 2)}=4 C L n\left[1+\frac{2 \beta}{K}\right]+\frac{4 \beta C}{5} \frac{1+5 \beta / K}{(1+2 \beta / K)^{2}}+\frac{4 C}{3}
$$

\section{3}

\section{Phase $\mathrm{A} 3$ : zones plastiques $\mathrm{PL}_{\mathrm{s}}$ ' $(R, \mathrm{x})$ et $\mathrm{PL}_{\mathrm{s}}(\mathrm{x}, \mathrm{w})$, zone élastique $\mathrm{EL}(\mathrm{w}, \infty)$}

Lorsque $w$ dépasse $\mathrm{x}, c^{\prime}$ est-à-dire lorsque $\Delta \mathrm{P}$ devient supérieur à $\Delta \mathrm{P}^{\left(\mathrm{A}^{2}\right)}$, la configuration évolue par rapport à la précédente : on a deux zones de plasticité du sol, une avec décollement à l'interface sol/boulon et l'autre avec adhérence parfaite.

Tous calculs faits, on montre que, pour cette phase A3, les équations liant les différents paramètres $\mathrm{X}, \mathrm{W}$ et $\Delta \mathrm{P}$ sont exactement les mêmes que celles de la phase A2.

\section{Condition de la fin de la phase A3}

La fin de cette troisième phase a lieu lorsque la limite élastique des boulons est atteinte, c'est-à-dire lorsque :

$$
\varepsilon_{r}^{b}=\varepsilon_{y b}=\frac{\sigma_{y b}}{E_{b}} \text { avec } \varepsilon_{r}^{b}=\left\{\frac{\frac{K C(r / R-1)}{\beta E_{s}}}{\frac{K C(X-1) X^{3}}{\beta E_{s}}\left(\frac{R}{r}\right)^{3}}\right\} \text { si } R<r<x
$$

Comme $\varepsilon_{r}^{b}$ est monotone croissante en r sur l'intervalle $[R, x]$ et décroissante sur l'intervalle $[x, \infty]$, cette condition est d'abord atteinte en son maximum c'està-dire en $x$. On a alors :

$\varepsilon_{y b}=\frac{K C(X-1)}{\beta \mathrm{E}_{5}}$

D'où la valeur particulière de $\mathrm{X}$, notée $\mathrm{X}^{(\mathrm{A} 3)}$, marquant l'entrée en plasticité des boulons :

$$
X^{(A 3)}=1+\frac{\Omega}{K}
$$

Cette valeur particulière correspond au seuil de chargement $\Delta \mathrm{P}^{(\mathrm{A} 3)}$ qui vérifie l'équation (40). Tous calculs faits, on obtient:

$$
\Delta P^{(A 3)}=\frac{4 C}{3}+\frac{4}{3} C \operatorname{Ln}\left[\frac{\Omega}{2 \beta}\left(1+\frac{\Omega}{K}\right)^{3}\right]+K C\left[1-\frac{8 \Omega / K^{+5}}{5(1+\Omega / K)^{2}}\right]
$$

\section{4}

\section{Phase $\mathrm{A} 4$ : zones plastiques $\mathrm{PL}_{5}(\mathrm{R}, \mathrm{x})$, $P L_{s} / P_{b}(x, z)$ et $P L_{s}(z, w)$, zone élastique $E[(W, \infty)$}

Le début de la plastification des boulons marque l'arrêt de la propagation de la zone de déchaussement entre le sol et le boulon. Le rayon de déchaussement $x$ atteint alors sa valeur maximale et ne varie plus. Il reste par la suite égal à $X^{(A 3)}=1+\frac{\Omega}{K}$.
Le déplacement est toujours donné par l'expression (20).

La continuité de la traction $\mathrm{T}_{\mathrm{b}}$ en $\mathrm{z}$ permet de déduire la constante $\mathrm{B}$ :

$B=\frac{\Omega}{2 \beta} \frac{C}{E_{s}} Z^{3}$

Dans la zone élastique EL, $\sigma$ admet toujours la même expression (22).

La continuité de $\mathrm{T}_{\mathrm{b}}$ en x conduit toujours à la même expression (23) de $\mathrm{B}$.

Dans la zone plastique PL'; l'expression (33) de $\sigma_{r}$ est toujours valable.

Dans la zone plastique $\mathrm{PL}_{5}$, le critère de plasticité du composite et l'équation d'équilibre (15) permettent de déduire :

$\sigma_{r}-\sigma_{\theta}=2 C+\sigma_{0}(r)=2 C+d_{b}\left(\frac{R}{r}\right)^{2} T_{b}=-\frac{r}{2} \frac{\partial \sigma_{r}}{\partial r}$

En remplaçant par l'expression (4) de $T_{b}$ (dans le cas $\varepsilon_{\mathrm{r}}<\varepsilon_{\mathrm{yb}}$ ) et en résolvant cette équation différentielle, on obtient:

$\sigma_{r}=-4 C \operatorname{Ln} \frac{r}{R}+\frac{4}{5} \beta E_{s} B \frac{R^{5}}{r^{5}}+D^{\prime} C$

où $D^{\prime}$ est une constante d'intégration sans dimension.

Dans la zone PLs/PL $L_{b}$ l'équation différentielle (48) est toujours valable mais l'expression (4) de $\mathrm{T}_{\mathrm{b}}$ est celle du $\operatorname{cas} \varepsilon_{\mathrm{r}}>\varepsilon_{\mathrm{yb}}$ (i.e. boulon plastifié). On obtient finalement

$\sigma_{r}=-4 C \operatorname{Ln} \frac{r}{R}+\Omega C\left(\frac{R}{r}\right)^{2}+D^{\prime \prime} C$

où D" est une autre constante d'intégration sans dimension.

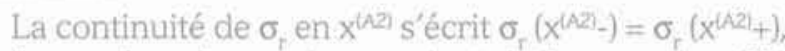
oủ le membre de gauche provient de l'expression (33) de $\sigma_{r}$ dans la zone PL, et celui de droite de l'expression (50) de $\sigma_{\mathrm{f}}$ dans la zone $\mathrm{PL}_{\mathrm{s}} / \mathrm{PL}_{\mathrm{b}}$. Ce qui donne, après simplification :

$D^{\prime \prime}=-\frac{P_{i}}{C}-\frac{\Omega K}{\Omega+K}$

De la même manière, la continuité de $\sigma_{r}$ en $z$, en utilisant les relations (48), (50) et (51) conduit à :

$D^{\prime}=-\frac{P_{i}}{C}-\frac{\Omega K}{\Omega+K}+\frac{3}{5} \frac{\Omega C}{Z^{2}}$

Enfin, la continuité de $\sigma_{r}$ en $w$, tenant compte de (22), (48) et (52), permet de déduire l'équation suivante:

$$
\frac{\Delta P}{C}-\frac{\Omega K}{\Omega+K}-4 \operatorname{Ln} W+\frac{3}{5} \frac{\Omega}{Z^{2}}-\frac{2 \Omega Z^{3}}{3 \beta W^{3}}=0
$$

La détermination des rayons inconnus $w$ et $z$ se déroule comme suit. La première équation vient du fait que le critère de plasticité du sol ??? est tout juste atteint en $w^{*}$. On retrouve alors l'expression (38) où on introduit la valeur (47) de B. D'où :

$W^{3}=\frac{\Omega}{2 \beta} Z^{3}$

La deuxième équation se déduit en remplaçant W dans l'équation (53) par la valeur ci-dessus; il en résulte l'équation suivante sur Z : 
$\Sigma_{4}(Z, \Delta P)=\frac{\Delta P}{C}-\frac{4}{3} \operatorname{Ln} \frac{\Omega Z^{3}}{2 \beta}+\frac{3}{5} \frac{\Omega}{Z^{2}}-\frac{\Omega K}{\Omega+K}-\frac{4}{3}=0$

Notons que le début de plastification de cette phase commence avec $\mathrm{Z}=1+\frac{\Omega}{\mathrm{K}}$.

On démontre facilement qu'on a $\Sigma_{4}(1+\Omega / K, \Delta \mathrm{P})>0$, $\Sigma_{4}(\infty, \Delta \mathrm{P})<0$ et $\Sigma_{4}^{\prime}(\mathrm{Z}>1+\Omega / K)<0$, l'équation ci-dessus admet donc une et une racine unique dans l'intervalle $(1+\Omega / K, \infty)$.

De plus, l'hypothèse prise au départ $(2 \beta \leq \Omega)$ fait que $z$ ne rattrape pas $w$, il n'y a donc pas d'autres phases.

\section{5}

\section{Conditions mathématiques caractérisant les trois cas A, B et C}

Dans ce paragraphe, on explicite les conditions d'inégalités mathématiques (données dans le tableau II) qui régissent chacun des trois cas.

Dans le traitement du cas A, la plastification du sol précède celle des boulons $\left(\bar{X}^{(A 1)} \leq X^{(B 1)}=1+\frac{\Omega}{K}\right)$.

D'autre part, la plastification des boulons intervient à l'intérieur de la zone où le sol est en plasticité, autrement dit, après le croisement des rayons w et $\mathrm{x}$; cette condition est traduite mathématiquement par l'inégalité $1+\frac{2 \beta}{K} \leq 1+\frac{\Omega}{K}$.

Pour le cas B, la plastification du sol précède également celle des boulons $\left(\bar{X}^{(A 1)} \leq 1+\frac{\Omega}{K}\right)$. En revanche, la plastification des boulons intervient dans la zone du comportement élastique du sol, soit avant le croisement des rayons $\mathrm{w}$ et $\mathrm{x}$. Mathématiquement, cette condition s'écrit $1+\frac{\Omega}{K} \leq 1+\frac{2 \beta}{K}$.

Contrairement aux cas $\mathrm{A}$ et $\mathrm{B}$, dans le cas $\mathrm{C}$ la plastification des boulons précède celle du sol $\left(1+\frac{\Omega}{K} \leq X^{(A 1)}\right)$.

L'inégalité $X^{(A 1)} \leq 1+\frac{2 \beta}{K}$ est quant à elle toujours valable ; elle découle de l'équation (30)

\section{7}

\section{Comparaison avec des résultats numériques}

On compare les résultats analytiques aux résultats numériques obtenus en utilisant un code de calcul tridimensionnel par différences finies nommé FLAC 3D (Coatzee et al., 1993).

Les paramètres géométriques et géomécaniques, ainsi que le phasage des travaux s'inspirent d'un projet réel de tunnel (la traversée de Toulon, France) pour le passage dans des terrains meubles de qualité médiocre (Dias et al., 1998).

\section{Modèle numérique et paramètres de calcul}

Dans les deux approches, on considère un tunnel profond circulaire de rayon $R=5,8 \mathrm{~m}$ creusé dans un massif supposé isotrope avec une pression géostatique initiale avant creusement P. Le sol est modélisé par un comportement élastoplastique parfait obéissant au critère de Tresca. La cohésion vaut $\mathrm{C}=0,2 \mathrm{kPa}$, le module d'Young est de $\mathrm{E}_{\mathrm{s}}=50 \mathrm{MPa}$.

La comparaison est menée suivant deux valeurs du facteur de charge $: N=\frac{P_{x}}{C}=\frac{\Delta P}{C}=4$ ou 6 .

Les boulons utilisés ont une section de $\mathrm{S}_{\mathrm{b}}=$ $1400 \mathrm{~mm}^{2}$, un module d'Young de $E_{\mathrm{p}}=20 \mathrm{GPa}$, une limite élastique de $\sigma_{\mathrm{vb}}=500 \mathrm{MPa}$ et leur nombre en paroi est pris égal à $N_{b}=52$ boulons. Leur longueur est supposée infinie dans l'approche analytique alors qu'elle est prise initialement égale à dix fois le diamètre du tunnel pour l'approche numérique.

La figure 6 montre le maillage 3D utilisé, qui tient compte de la symétrie du problème.

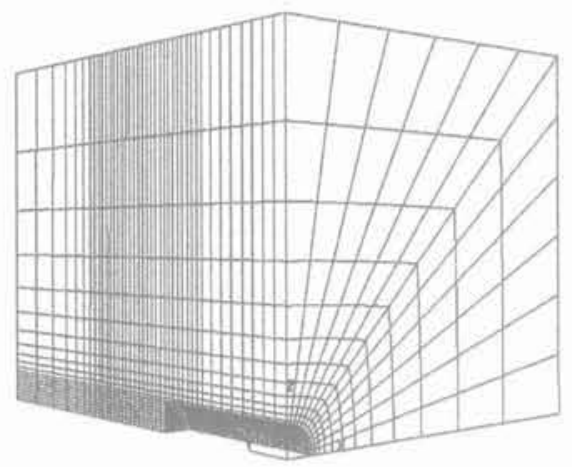

FIG.

Maillage tridimensionnel du modèle numérique,

Three dimensional mesh of the numerical model.

TABLEAU III Caractéristiques du revêtement béton (cas de l'approche numérique).

$\begin{array}{lc}\text { Module élastique E (MPa) } & 10000 \\ \text { Coefficient de poisson } & 0,2 \\ \text { Epaisseur (m) } & 0,22 \\ \text { Densité }\left(\mathrm{kg} / \mathrm{m}^{2}\right) & 2500\end{array}$

Pour l'approche numérique, l'excavation se déroule en 12 phases de $3 \mathrm{~m}$ chacune ; on suppose que le revêtement en béton du tunnel, dont les caractéristiques sont données dans le tableau III, a un comportement élastique linéaire et que sa pose a lieu au fur et à mesure de l'excavation, ce qui simule bien l'usage courant de prévoûtes dans la réalité.

La figure 7 présente la répartition des boulons au front et la figure 8 la modélisation quant aux boulons et l'interface avec le terrain. 


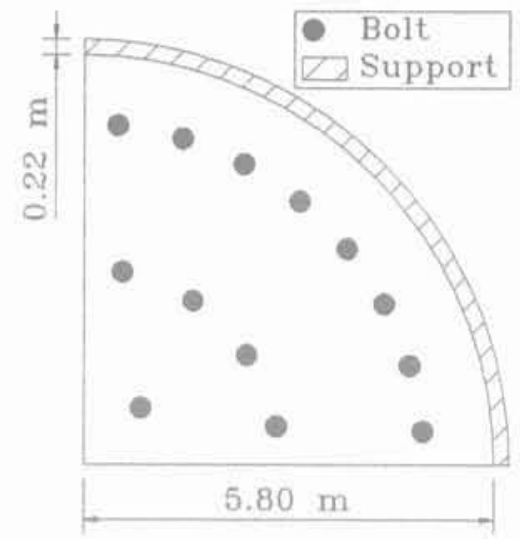

FIG.7 Répartition des boulons au front (52 au total).

Emplacement of bolts at tunnel face ( 52 in total).

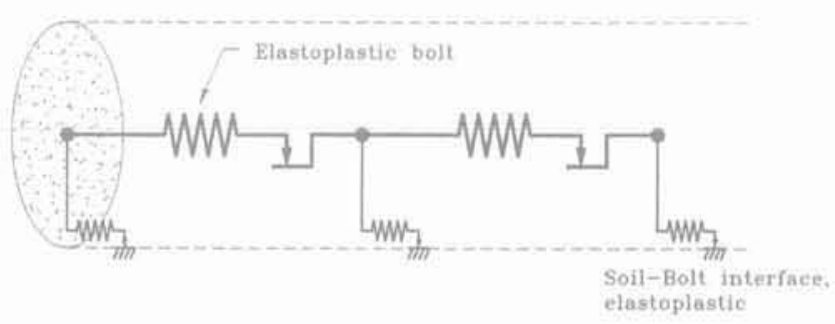

FIG. 8 Modélisation des boulons et du comportement d'interface sol/boulon.

Modeiling of the behaviours of bolts and the bolt-ground interface.

\section{Résultats et commentaires}

La comparaison est menée sur la répartition de la traction le long d'un boulon et la valeur de l'extrusion au front, pour quatre valeurs de la résistance à l'arrachement $\mathrm{R}_{5}(\mathrm{kN} / \mathrm{ml}): 25,60,100$ et l'infini (i.e, adhérence parfaite).

En ce qui concerne l'extrusion au front, signalons que dans le cas de l'approche numérique, elle n'est pas constante mais varie le long de la paroi frontale (de 0 à R) alors que dans le cas de l'approche analytique, on calcule un déplacement moyen. Afin de les comparer, les valeurs d'extrusions obtenues numériquement ont donc été moyennées sur la surface totale du front du tunnel. 12.

Les résultats obtenus sont donnés par les figures 9 à

Au vu de ces résultats, on constate que le nouveau modèle analytique développé dans cet article prend bien en compte la mobilisation progressive de la traction des boulons à partir du front (sans plaque d'ancrage), et l'existence d'un pic à l'intérieur du massif (Figs. 9 et 10), ce qui n'est pas le cas avec le modèle de base à adhérence parfaite. Ainsi, l'estimation de la traction maximale est réellement meilleure : l'écart maximal entre les résultats numériques et analytiques avec adhérence non parfaite étant seulement de $5 \%$ (courbes $\mathrm{c}$ et $\mathrm{g}: \mathrm{N}=6$ et $\mathrm{R}_{\mathrm{n}}=100 \mathrm{kN} / \mathrm{ml}$ ), alors que la comparaison avec les résultats analytiques à adhérence

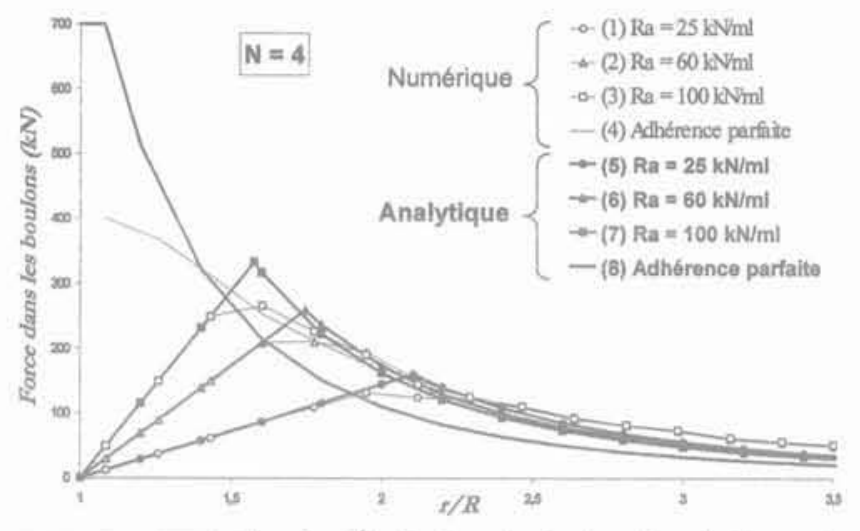

FIG.9 $\mathrm{N}=4-$ Évolution de la traction le long du boulon pour différentes valeurs de la résistance à l'arrachement : comparaison entre modèle analytique et modèle numérique,

$\mathrm{N}=4$ - Evolution of the bolt tension profile for different pull-out strengths : comparison between analytical and numerical model.

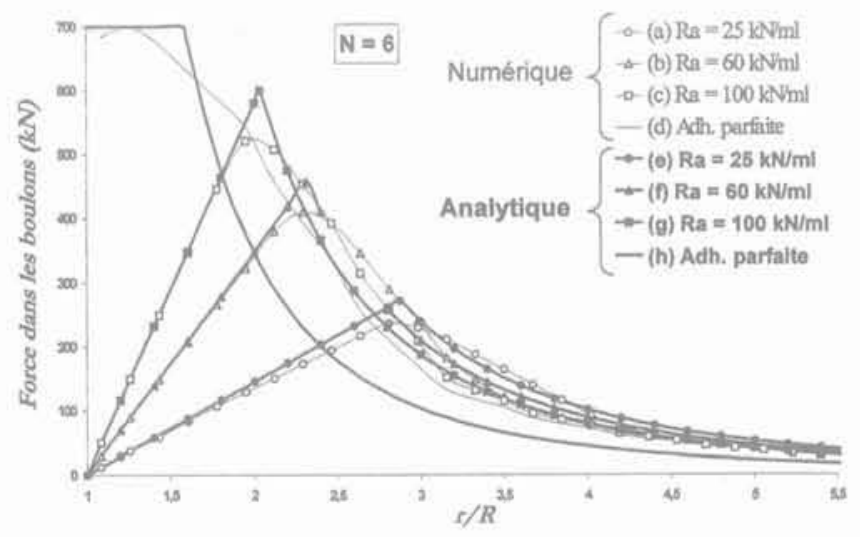

FG. $10 \mathrm{~N}=6$ - Évolution de la traction le long du boulon pour différentes valeurs de la résistance à l'arrachement : comparaison entre modèle analytique et modèle numérique.

$\mathrm{N}=6$-Evolution of the bolt tension profile for different pull-out strengths : comparison between analytical and numerical model.

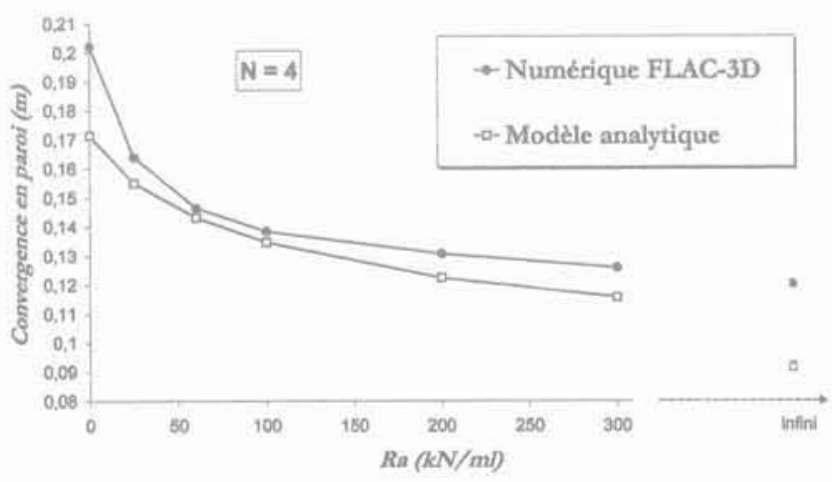

FIG. $11 \mathrm{~N}=4-$ Extrusion en paroi en fonction de la résistance à l'arrachement : comparaison entre modèle analytique et modèle numérique.

$\mathrm{N}=4$ - Face extrusion vs, pull-out strength: comparison between analytical and numerical model. 


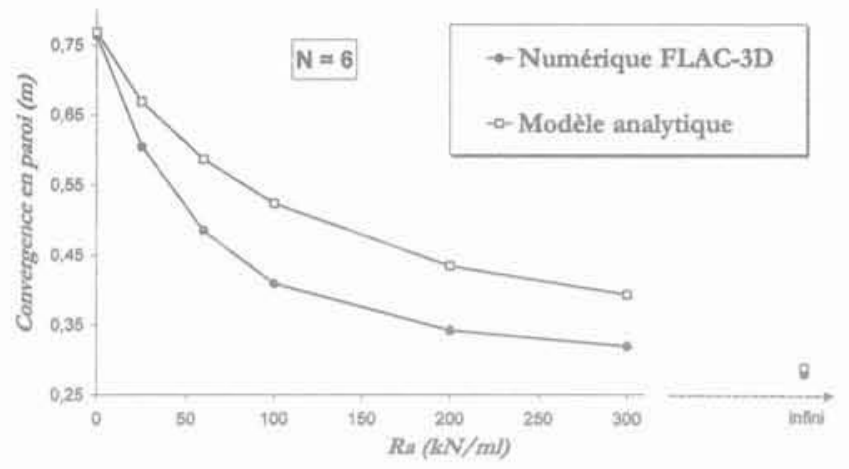

FIG. $12 \mathrm{~N}=6$ - Extrusion en paroi en fonction de la résistance à l'arrachement : comparaison entre modèle analytique et modèle numérique.

$\mathrm{N}=6$ - Face extrusion vs, pull-out strength comparison between analytical and numerica. model.

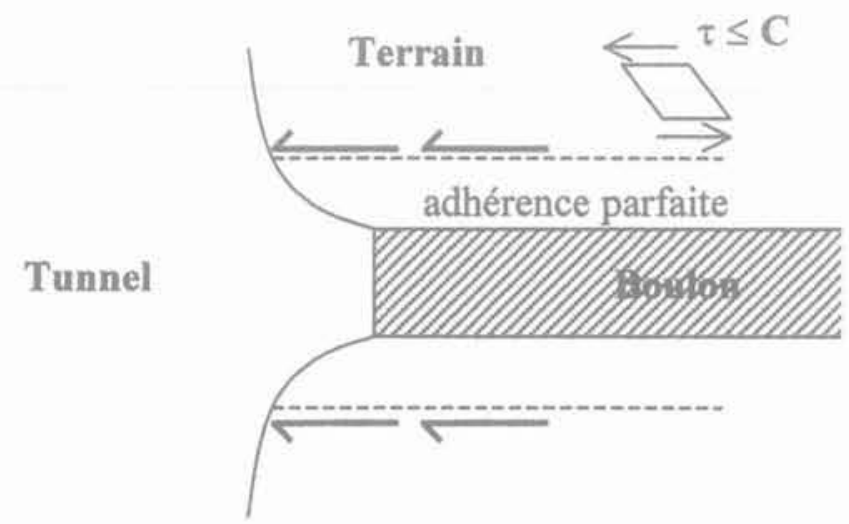

FG. 13 Cas réel : limitation de la contrainte de cisaillement à l'interface boulon/sol par la cohésion du sol.

Real situation : upper bound of the shear stress at bolt-ground interface by the ground cohesion.

parfaite (courbe h) donne une surévaluation de la traction maximale de plus de $150 \%$ pour le cas $\mathrm{N}=6$ et $\mathrm{R}=$ $25 \mathrm{kN} / \mathrm{ml}$ (courbe a).

Au-delà de la valeur maximale, l'allure générale de la répartition de la traction dans le boulon présente également une très bonne concordance comme le montrent bien les figures 9 et 10. Encore une fois, cela met en évidence le rôle important joué par le phénomène de l'adhérence non parfaite : le développement analytique présenté dans cet article est donc nécessaire.

Remarquons que, lors de la mise en place du boulonnage frontal sur chantier, même si l'injection est bien faite, assurant un bon contact entre le scellement du boulon et le sol (i.e. adhérence $\alpha$ parfaite $)$ à l'interface), la résistance du sol (caractérisée par sa cohésion C) limite la contrainte de cisaillement au sein de celui-ci; un glissement intervient dès lors que la contrainte de cisaillement dans le sol atteint sa cohésion. Par conséquent, la cohésion du sol pose une limite supérieure physique à la résistance à l'arrachement du boulon, indépendamment de la qualité du scellement (donc de l'adhérence limite $\mathrm{q}_{5}$ ).

\section{8}

\section{Retour d'expérience in situ}

En février 1996 a débuté le chantier du tunnel de Tartaiguille, l'un des premiers en France utilisant la technique du boulonnage du front de taille. A cause de problèmes de convergence et de chute de blocs (EMMC, 1998), de nombreux essais d'auscultation (convergence en paroi et extrusion du front) ont été réalisés tout au long du tunnel. Outre la richesse et l'excellente qualité des mesures, différentes campagnes de reconnaissance géotechnique ont également été effectuées permettant d'avoir une bonne appréciation des paramètres du sol.

\section{1}

\section{Présentation générale du chantier}

Le tunnel de Tartaiguille, réalisé près de Montélimar (France), s'étend sur une longueur de $2338 \mathrm{~m}$ en souterrain, sa couverture étant supérieure à $75 \mathrm{~m}$ sur $1600 \mathrm{~m}$ et atteignant au maximum $137 \mathrm{~m}$. L'excavation a débuté simultanément par les deux extrémités. Les différentes formations géologiques rencontrées sont présentées à la figure 14. La section du tunnel excavé est estimée à $\mathrm{S}=180 \mathrm{~m}^{2}$ ce qui correspond à un rayon moyen de $\mathrm{R}=7,4 \mathrm{~m}$. L'utilisation du boulonnage frontal en pleine section concerne le creusement dans les argiles marneuses du stampien inférieur entre les PM 495 et 1370 .

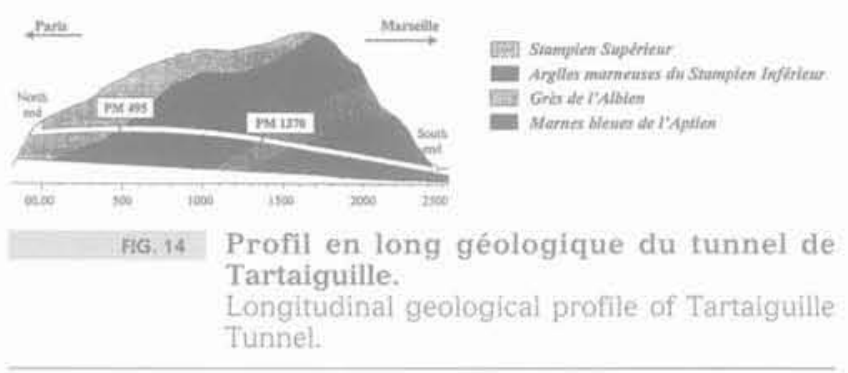

Caractéristiques du sol (valeurs du comité de pilotage)

Dans le cadre de l'étude de faisabilité de la méthode d'excavation en pleine section, de nouveaux essais ont été réalisés : essai au dilatomètre (vertical, horizontal), essai à la plaque $\varnothing 60$, essai au pressiomètre autoforeur... En raison d'une grande dispersion de ces résultats, on a choisi d'utiliser comme paramètres du sol ceux retenus par le comité de pilotage du chantier rappelés dans le tableau IV.

TABIEAUIN Paramètres de calcul retenus.

\begin{tabular}{ll}
\hline Module d'Young & $E=400 \mathrm{MPa}$ \\
Cohésion & $\mathrm{C}_{\mathrm{u}}=1,2 \mathrm{MPa}$ \\
Angle de frottement & $\varphi=0^{\circ}$ \\
Poids volumique & $\gamma=21 \mathrm{kN} / \mathrm{m}^{3}$ \\
\hline
\end{tabular}




\section{Extrusomètres}

Les données brutes de plusieurs extrusomètres (Jassionnesse, 1998) ont été récupérées et, après exploitation, il en ressort pour chacun plusieurs profils d'extrusion frontale du massif correspondant chacun à la perturbation due au creusement entre des intervalles différents. Afin d'apprécier le degré de précision de ce modèle et de délimiter son domaine de validité, il apparaît intéressant de comparer ces profils à ceux obtenus à l'aide du modèle analytique. La confrontation des résultats est menée seulement à partir de deux extrusomètres $\left(n^{\circ} 1\right.$ et $\left.n^{\circ} 2\right)$, les observations in situ montrant que les autres étaient situés dans un terrain fortement hétérogène (Wong et al., 1999).

Ces extrusomètres se situant à une profondeur d'environ $\mathrm{H}=110 \mathrm{~m}$, la pression géostatique vaut donc $\mathrm{P}_{\mathrm{f}}=2,3 \mathrm{MPa}$. Les boulons utilisés pour ce chantier ont lés caractéristiques suivantes : $S_{b}=840 \mathrm{~mm}^{2}, E_{k}=$ $40 \mathrm{GPa}, \sigma_{\mathrm{yb}}=1000 \mathrm{MPa}$. Le nombre de boulons mis en place en paroi vaut $N_{b}^{1}=123$ pour l'extrusomètre $n^{\circ} 1$ et $N_{b}{ }^{2}=153$ pour le $n^{\circ} 2$, soient des densités $d_{b}{ }^{2}=0,715$ et $\mathrm{d}^{2}=0,889$ boulon $/ \mathrm{m}^{2}$. A défaut de connaitre la valeur réelle du seuil de contrainte de scellement $\mathrm{q}_{\mathrm{s}}$ (issue par exemple des résultas d'essais d'arrachement in situ), on la prend égale à la cohésion du sol (\$ 7.2). Le diamètre du forage (diamètre du cylindre d'interface scellementterrain) vaut $D_{\text {scetl }}=10 \mathrm{~cm}$; d'où une valeur estimative de la résistance a l'arrachement du boulon : $R_{a}=\pi D_{\text {scell }}$ $\mathrm{C}=377 \mathrm{kN} / \mathrm{ml}$. (Cf. formule (5)).

\section{2}

\section{Confrontation des résultats et commentaires}

Les résultats obtenus après confrontation entre les prévisions analytiques et les données in situ recueillies par les deux extrusomètres sont présentés sur les figures 15 et 16 .

Remarquons pour commencer que les profils d'extrusion mesurés in situ forment une famille de courbes se situant dans une zone très étroite montrant une excellente cohérence des mesures. En effet, la valeur d'extrusion en paroi est comprise entre 25 et $30 \mathrm{~mm}$ (soit $20 \%$ d'écart entre la valeur minimale et la valeur maximale) pour l'extrusomètre $n^{\circ} 1$, et elle est d'environ $30 \mathrm{~mm}$ (avec $5 \%$ de dispersion) pour l'extrusomètre $n^{\circ} 2$. Ceci met en évidence une bonne homogénéité du terrain dans le tronçon considéré ; les conditions in situ se rapprochent donc bien des hypothèses faites sur le modèle théorique. De plus, l'allure générale de la courbe d'extrusion qui peut être tirée de la superposition des courbes a la même tendance pour les deux extrusomètres. Enfin, l'analyse de ces profils d'extrusion montre l'existence d'une zone critique, s'étendant sur une distance de l'ordre du diamètre du tunnel, au-delà de laquelle le massif ne semble pas perturbé par l'excavation. Cette observation est cohérente avec le retour d'expérience général.

Au niveau de la comparaison, les prédictions d'extrusions du modèle sont remarquables puisqu'elles sont très proches de celles réellement observées. En effet, le calcul avec les paramètres les plus probables, selon le comité de pilotage donne une extrusion en paroi d'environ $32 \mathrm{~mm}$ pour les deux cas de figure considérés, soit une erreur relative maximale inférieure à $25 \%$. De plus, l'allure de la décroissance est assez proche de la forme prédite par le modèle, du moins à

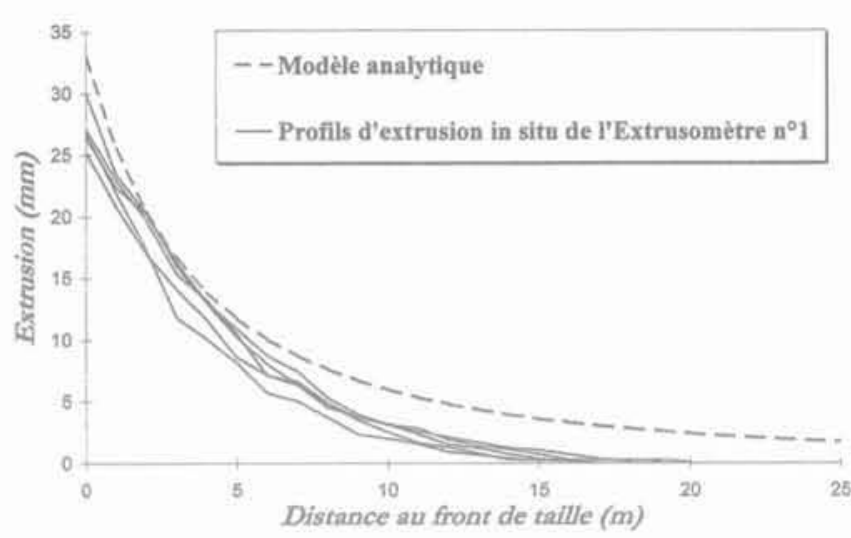

FIG. 15 Confrontation des résultats entre les mesures de l'extrusomètre $n^{\circ} 1$ et le modèle analytique.

Comparason between in situ data from extrusometer $n^{\circ} 1$ and the analytical model.

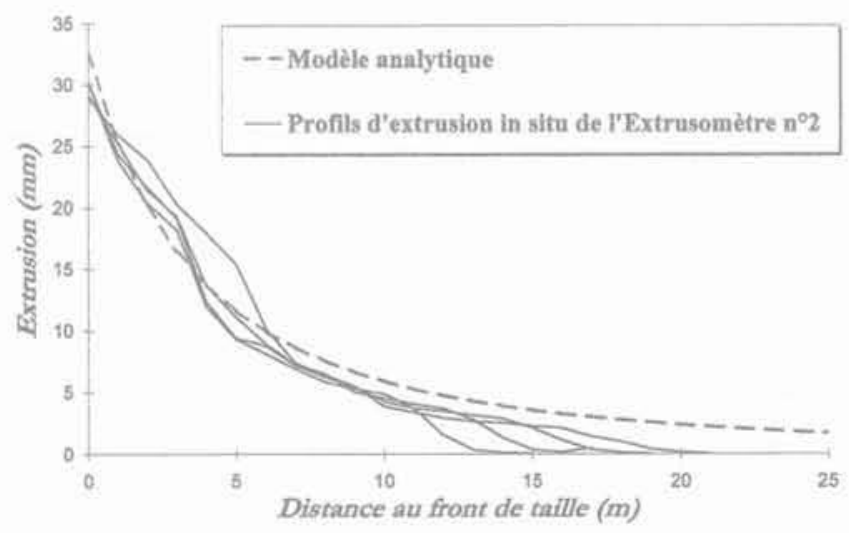

FIG. 16 Confrontation des résultats entre les mesures de l'extrusomètre $n^{\circ} 2$ et le modèle analytique,

Comparison between in situ data from extrusometer $n^{\circ} 2$ and the analytical model.

Tintérieur de la zone d'influence. Au-delà de cette distance, une divergence commence à apparaitre. La nullité de l'extrusion n'est théoriquement atteinte qu'à l'infini en raison des hypothèses inhérentes à ce genre de modèles analytiques simplifiés alors que l'extrusion mesurée est par hypothèse prise comme nulle au point d'ancrage. Néanmoins, n'étant plus dans la zone critique, cette divergence n'a aucune conséquence pratique.

Il convient toutefois de se garder d'une généralisation immédiate de la portée de cette première comparaison, réalisée pour un site spécifique avec un jeu de caractéristiques géomécaniques particulières. A cela s'ajoute les difficultés habituelles dans l'interprétation des campagnes de reconnaissance et les dispersions usuelles des résultats de mesures. Une partie de ces difficultés provient d'un certain degré d'hétérogénéité (" naturelle ») du terrain in situ. Davantage d'observations de chantiers réels sont donc nécessaires pour mieux comprendre toutes les finesses du comportement d'un tel ouvrage et affiner les conclusions de cette première étude, et éventuellement apporter des améliorations au modèle théorique. Dans cet objectif, les essais en laboratoire avec un matériau artificiel homogène sous conditions contrôlées pourront compléter 
avantageusement les observations in situ. C'est pourquoi une étude expérimentale sur modèle réduit sous gravité terrestre est actuellement en cours de réalisation. Les résultats attendus devraient permettre de quantifier, grâce à une instrumentation appropriée, les mouvements à l'intérieur du massif, sous conditions contrôlées, et ainsi d'apprécier l'efficacité réelle des boulons.

\section{9}

\section{Abaques de prédimensionnement}

Les bons résultats des comparaisons menées avec un calcul numérique et des données in situ donnent une certaine confiance dans la validité et donc l'applicabilité de notre modèle théorique. C'est pourquoi différents abaques de prédimensionnement du boulonnage sont ici présentés montrant l'utilité du modèle.

Les figures 17 à 19 permettent un prédimensionnement rapide du boulonnage au front de taille en considérant que l'on veuille rester dans le domaine élastique des boulons, pour trois valeurs du paramètre $\mathrm{K}(1.5,2.5$ et 4).

Les courbes pleines sont tracées à $\mathrm{P}^{*}$ fixé, en supposant que $\Omega$ est suffisamment grand pour que les boulons restent indéfiniment élastiques. Celles-ci sont donc valables tant que la limite élastique des boulons $\sigma_{\mathrm{yb}}$ n'est pas atteinte, c'est-à-dire tant que l'on se place à gauche de la courbe pointillée pour $\Omega$ donné. En effet, ces dernières représentent la valeur de la convergence lorsque les boulons plastifient.

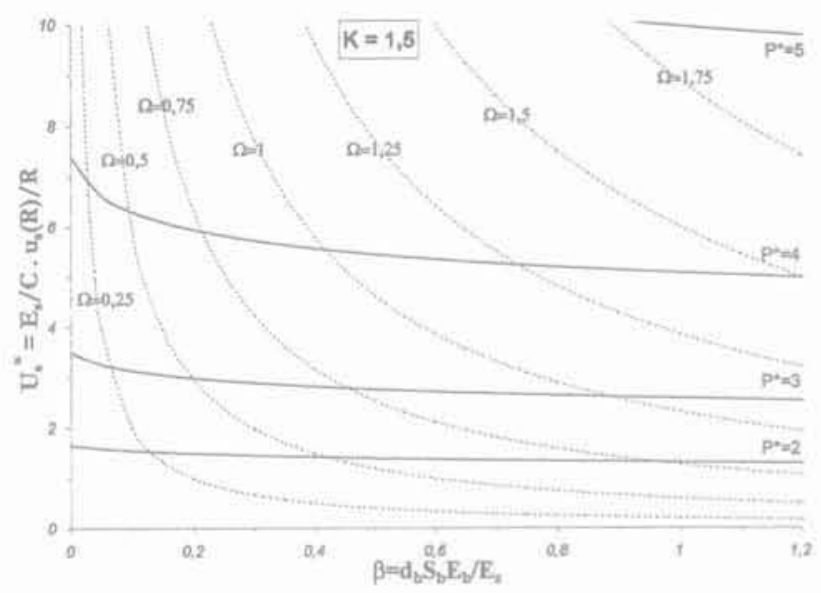

FIG. 17 Abaque de prédimensionnement du boulonnage pour $\mathrm{K}=1,5$.

Preliminary design chart of bolts for $K=1,5$.

Le principe d'utilisation de tels abaques est illustré par l'exemple qui suit. Pour un projet de tunnel de rayon $R=5 \mathrm{~m}$ creusé dans un sol tel que $\mathrm{E}_{\mathrm{s}}=200 \mathrm{MPa}$ et $\mathrm{C}=200 \mathrm{kPa}$ à une profondeur $\mathrm{H}=40 \mathrm{~m}$ (soit $\mathrm{P}^{*}=\gamma \mathrm{H} / \mathrm{C}=$ 4 , en supposant que $\gamma=20 \mathrm{kN} / \mathrm{m}^{3}$ ), on désire limiter la valeur de la convergence moyenne en paroi à $3 \mathrm{~cm}$ (i.e.) $\varepsilon_{\text {parol }}=1,2 \%$ ), ce qui correspond à $U^{*}=\frac{E_{s}}{C} \frac{u(R)}{R}=6$, afin d'éviter tout tassement important en surface. En prenant une résistance à l'arrachement du boulon égale à $R_{a}=100 \mathrm{kN} / \mathrm{ml}$ et une densité de boulonnage $d_{b}=1$ boulon/m, alors on obtient $\mathrm{K}=2,5$. Ces valeurs de $\mathrm{U}^{*}, \mathrm{P}^{*}$ et $\mathrm{K}$ correspondent au point $\pi$ sur l'abaque de la figure 18 .

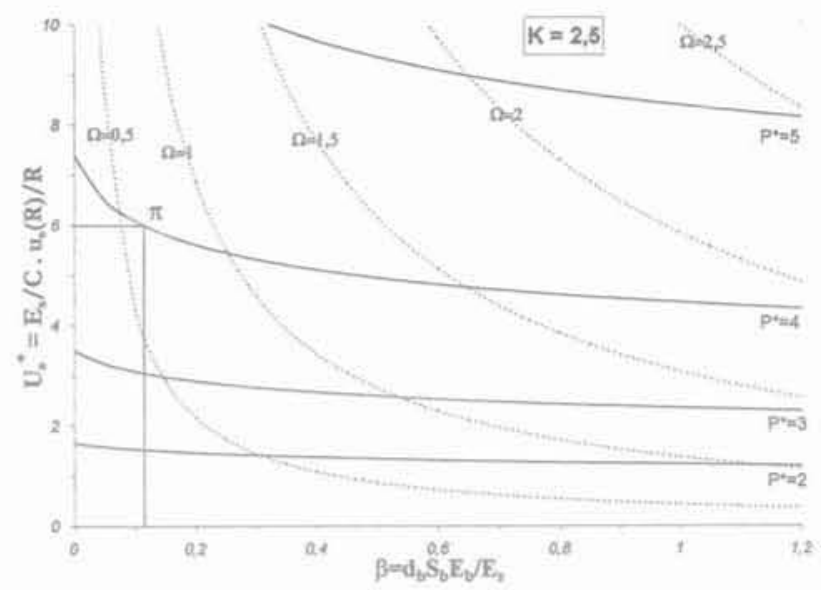

Fig. 18 Abaque de prédimensionnement du boulonnage pour $\mathrm{K}=2,5$. Preliminary design chart of bolts for $\mathrm{K}=2,5$.

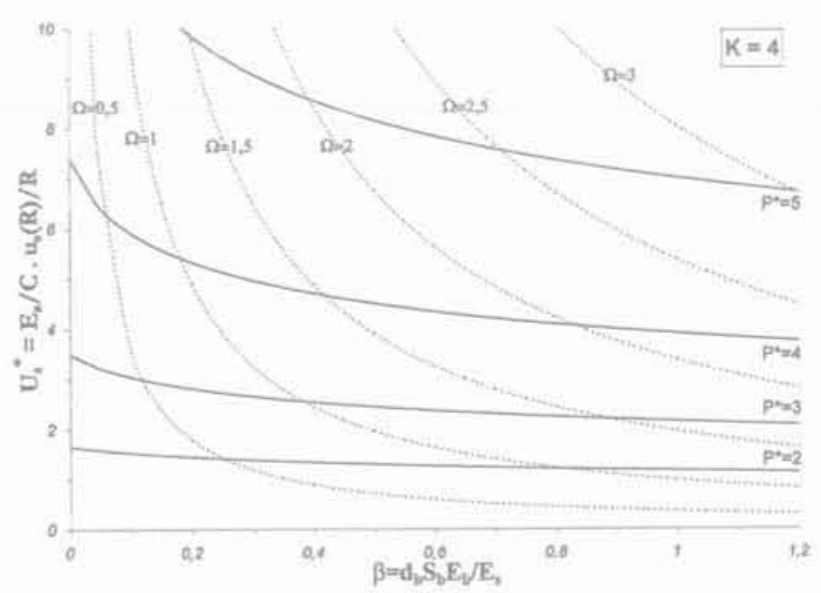

Fig. 19 Abaque de prédimensionnement du boulonnage pour $\mathrm{K}=4$.

Preliminary design chart of bolts for $\mathrm{K}=4$.

D'où la valeur minimale de $\beta=0,13$. Cette condition est satisfaite pour un boulonnage vérifiant $S_{b}=1400 \mathrm{~mm}$, $E_{b}=20 \mathrm{GPa}$ et $\sigma_{v b}=500 \mathrm{MPa}$. Ces paramètres fixent également la valeur de $\Omega$, ici 3,5 . Le point $\pi$ étant bien à gauche de la courbe pour laquelle $\Omega=3,5$, les boulons restent donc élastiques pour le cas de chargement considéré.

\section{0}

\section{Conclusion}

Pour compléter les approches types « stabilité », un modèle théorique original sur le comportement du front de taille d'un tunnel renforcé par du boulonnage a été proposé, en s'appuyant sur de précédents travaux. Ce nouveau modèle permet de prendre en compte une limite d'adhérence de l'interface boulon/sol, qui pose une limite supérieure à l'effet de renforcement du boulonnage. Ceci permet une meilleure évaluation de la traction (valeur maximale et répartition) du boulon, et par conséquence du champ de déplacement du massif, qui sont deux paramètres essentiels pour le dimension- 


\begin{tabular}{|c|c|c|}
\hline & $\left.\operatorname{Cas} B: X^{\prime} 1\right)<1+\Omega / K<1+2 \beta / K$ & $\operatorname{Cas} C: \mathrm{X}^{(A 1)} \rightarrow 1+\Omega / \mathrm{K}$ \\
\hline Seuils de chargement & $\begin{array}{l}\Delta P^{(A+1)}=\Delta P^{(A)} \\
\Delta P^{(B 2)}=\Delta P^{(n) 3} \\
\Delta P^{(B 3)}=\frac{4 C}{3}+4 C \operatorname{Ln}\left[1+\frac{\Omega}{K}\right]+\frac{\Omega K C}{\Omega+K}\left[1-\frac{3}{5} \frac{\Omega^{25} K}{(2 \beta)^{20}(\Omega+K)} .\right.\end{array}$ & $\begin{array}{l}\Delta P^{(i)}=\frac{\Omega C K^{2}}{(\Omega+K)^{2}}\left\lfloor\frac{2}{3 \beta} \frac{(\Omega+K)^{3}}{K^{3}}+\frac{\Omega}{K}+\frac{2}{5}\right\rfloor \\
\Delta P^{\left(c^{2}\right)}=\frac{4 C}{3}+\frac{\Omega C K}{\Omega+K}-\frac{3 \Omega^{5} C}{5(2 \beta)^{2 /}} \\
\Delta P^{(C ;)}=\Delta P^{\text {(Bas) }}\end{array}$ \\
\hline $\begin{array}{l}\text { Phase B1/C1 } \\
0<\Delta^{P}<\Delta P^{(1)}\end{array}$ & \multicolumn{2}{|c|}{$\begin{array}{l}\frac{\Delta P}{C}=\frac{K(X-1)}{X ?}\left[\frac{2}{3} \frac{X}{\beta}+X+\frac{3}{5}\right] \\
U(R)=-\frac{C R K}{2 \beta E_{p}}(X-1) X^{3} \\
T_{b}^{\max }=\frac{C}{d_{b}} K(X-1) X^{3} \text { en } X\end{array}$} \\
\hline $\begin{array}{l}\text { Phase } B 2 / C 2 \\
\Delta P^{n i l} \leq \Delta P<\Delta P a i\end{array}$ & $\begin{array}{l}\frac{\Delta P}{C}=\frac{4}{3}+\frac{4}{3} \operatorname{Ln}\left[\frac{K X^{2}(X-1)}{2 \beta}\right]+K\left[1+\frac{3-8 X}{5 X^{2}}\right] \\
W^{3}=\frac{K}{2 \beta}(X-1) X^{3} \\
U(R)=-\frac{C R K}{2 \beta E_{n}}(X-1) X^{3} \\
T_{D}^{\text {max }}=\frac{C}{d_{b}} K(X-1) X^{3} \text { en } X\end{array}$ & $\begin{array}{l}\frac{\Delta P}{C}=\frac{2 \Omega}{3 \beta} Z^{\alpha}+\frac{\Omega K}{\Omega+K}-\frac{3 \Omega}{5} Z^{2} \\
X=1+\frac{\Omega}{K} \\
U(R)=-\frac{\Omega C R}{2 \beta E_{x}} Z^{3} \\
T_{b}^{\max }=\frac{\Omega C}{d_{b}} \quad \text { dans la zone } \mathrm{PL}_{b}\end{array}$ \\
\hline $\begin{array}{l}\text { Phase } B 3 / C 3 \\
\Delta P^{(2)} \leq \Delta P<\Delta P \text { io }\end{array}$ & \multicolumn{2}{|c|}{$\begin{array}{l}\frac{\Delta P}{C}=\frac{4}{3}+\frac{4}{3} L\left[\frac{\Omega Z^{3}}{2 \beta}\right]-\frac{3}{5} \frac{\Omega}{Z^{2}}+\frac{\Omega K}{\Omega+K} \\
X=1+\frac{\Omega}{K} \quad W^{3}=\frac{\Omega}{2 \beta} Z^{3} \\
U(R)=-\frac{\Omega C R}{2 \beta E_{b}} Z^{3} \\
T_{b}^{\max }=\frac{\Omega C}{d_{b}} \text { dans la zone PLi }\end{array}$} \\
\hline $\begin{array}{l}\text { Phase } B 4 / C 4 \\
\Delta P^{(3)} \leq \Delta P<P_{x}\end{array}$ & \multicolumn{2}{|c|}{ Mêmes expressions que pour la phase $\mathrm{B} 3 / \mathrm{C} 3$ sauf que la traction maximale est dans la zone $\mathrm{PL} / \mathrm{PI}$} \\
\hline
\end{tabular}

nement de l'ouvrage. Malgré cette complexité supplémentaire, une solution analytique a pu être établie, préservant ainsi tous les avantages liés au caractère explicite de la solution (voir par exemple Bérest, 1989).

Afin de mieux apprécier le domaine de validité du modèle proposé, ses prédictions sont confrontées d'abord à celles obtenues par une approche numérique tridimensionnelle, où chacun des boulons est modélisé individuellement par des éléments du type linéique avec prise en compte d'un comportement d'interface élastique parfaitement plastique. Les comparaisons sont ensuite menées avec des mesures in situ en provenance d'un chantier réel en cours de construction (au moment où l'étude est réalisée).

Sans oublier de préciser la portée encore limitée de cette première étude comparative, le haut degré de concordance obtenu est très encourageant, et incite à poursuivre ces travaux de recherche. Cette validation, bien qu'elle soit encore partielle, donne de la confiance dans l'applicabilité du modèle proposé, et confirme son 
utilité en tant qu'outil de prédimensionnement, très précieux au stade préliminaire de l'étude. En effet, sa performance (calcul quasi instantané) le rend particulièrement apte à effectuer des études de sensibilité des paramètres, avant de procéder à un dimensionnement précis et détaillé à l'aide des calculs numériques plus complexes et plus coûteux.

Enfin, soulignons que le modèle analytique proposé ici fait partie d'une démarche globale du problème faisant intervenir quatre types d'approches:

- modèles théoriques :

- modèles numériques :

- retour d'expériences in situ;

- expérimentation en laboratoire,

visant in fine à une meilleure compréhension du comportement de l'ouvrage et à un aboutissement des outils et règles de dimensionnement fiables, Ces approches font l'objet des travaux de recherche concertes entre les deux instituts cosignataires de la présente publication et le Centre d'études des tunnels (CETU).

\section{REMERCIEMENTS}

Les auteurs voudraient remercier Monsieur P. Dubois du CETL pour les discussions intéressantes et son soutient lors du démarrage de cette série de travaux de recherche.

\section{Annexe : résultats principaux des cas B et C}

Les scénarios (séquence d'apparition des zones plastiques) des cas B et $\mathrm{C}$ sont résumés dans les deux figures suivantes.

Les expressions principales (pressions critiques marquant le passage d'une phase à l'autre, équations définissant l'évolution des rayons $\mathrm{x}, \mathrm{w}$ et $\mathrm{z}$ ) sont don- nées dans le tableau $\mathrm{V}$ ainsi que, pour chaque phase, les valeurs de la convergence en paroi $U(R)$ et de la traction maximale $\mathrm{T}_{\mathrm{b}}^{\max }$ dans le boulon.

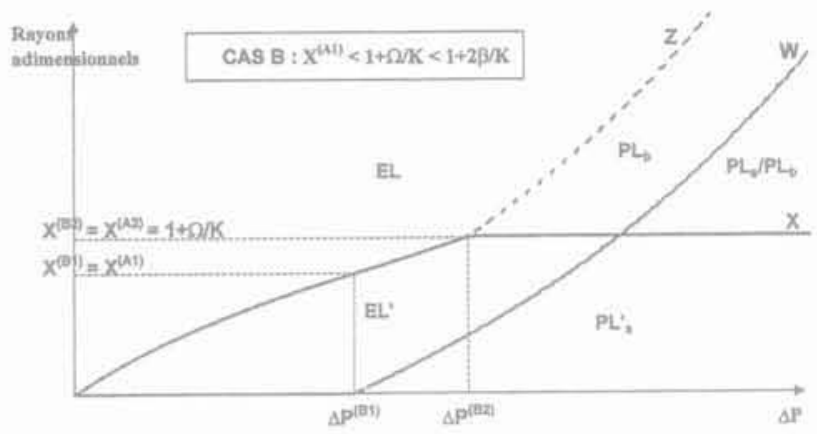

FIG.A1 CAS B - Évolution des rayons adimensionnels $\mathrm{X}, \mathrm{W}$ et $\mathrm{Z}$ en fonction du chargement $\triangle P$.

CASE B - Evolution of the dimensionless radi $\mathrm{X}, \mathrm{W}$ and $\mathrm{Z}$ vs, loading parameter $\Delta \mathrm{P}$

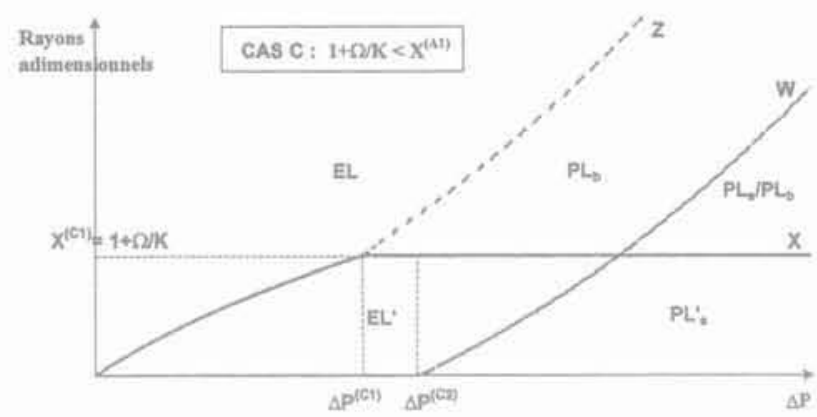

FG. AR CAS C - Evolution des rayons adimensionnels $\mathrm{X}, \mathrm{W}$ et $\mathrm{Z}$ en fonction du chargement $\triangle \mathrm{P}$.

CASE C - Evolution of the dimensionless radii $\mathrm{X}, \mathrm{W}$ and $\mathrm{Z}$ vs. loading parameter $\Delta \mathrm{P}$. 
Bérest P. - " Problèmes de mécanique associés au stockage souterrain. $n$ Doctorate Thesis, École nationale supérieure des mines de Paris, 1989, 361 p.

Coatzee M.J., Hart R.D., Varona P.M., Cundall A. - FLAC Basics, Itasca, Minneapolis, 1993.

Dias D., Subrin D., Wong H., Dubois P., Kastner R. - " Behaviour of a tunnel face reinforced by bolts : comparison between analytical - numerical models, y The Geotechnics of Hard Soils - Soft Rocks, Proc, II ${ }^{\text {nd }}$ Int. Conf., Napoli 12 14 oct. 1998, p. $961-972$.

E.M.M.C. - « Tartaiguille : une nouvelle méthode $n$, Tunnels et Ouvrages Souterrains, vol. 145, jan.-fév. 1998, p. 29-31.

Egger P., Subrin D., Wong H. - « Behaviour of a tunnel head reinforced by bolting : experimental study and theoretical modelling. 1) Proc, 9th Int. Congr. Soc: Rock. Mech., Paris, 1999.

Greuell E, - «Étude du soutènement des tunnels par boulons passifs dans les sols et les roches tendres par une méthode d'homogénéisation. » Doctorate Thesis École polytechnique, Palaiseau, 1993.

Indraratna $\mathrm{B}_{-}-\approx$ Effect of bolts on failure modes near tunnel openings in soft rock ग. Geotechnique, vol. 43, 1993, p. $433-442$.

Indraratna B., Kaiser P.K. - " Analytical model for the design of grouted roch bolts. I Int. J. Num. \& Anal. Methods in Geomech., vol. 14, 1990, p. 227-251.

Jassionnesse C. - " Contrôle de la déformation du massif renforcé par boulonnage au front de taille d'un tunne! Étude d'un cas réel en site urbain exploitation des mesures et modélisation par une méthode d'homogénéisation. y Doctorate Thesis, Institut national des sciences appliquées, Lyon, 1998, $233 \mathrm{p}$.
Jassionnesse C., Dubois P., Saïtta A. «Tunnel face reinforcement by bolting, soil bolts homogenization strain approach. „ Proc. Symp. On Geotechni. cal Aspects of Underground Constructions in Soft Ground, London. 15. 17 April 1996, Ed. Balkema Rotterdam. p. $373-378$.

Labiousse V. - Etude par convergenceconfinement du boulonnage à ancrage ponctuel comme soutènement de tunnels profonds creusés dans la roche. x. Revue française de Géotechnique, vol. 65 , 1994, p. 17-28.

Lunardi P. - " Fiber-glass tubes to stabilise the face of tunnels in difficult cohesive soils. „ SAIE - Seminar The Application of Fiber Reinforced Plastics (FRP) in civil structural engineering, Bologna, Italy, 1993.

Lunardi P. - « Conception et exécution des tunnels d'après l'analyse des déformations contrôlées dans les roches et dans les sols : présoutènement et préconfinement. x) Revue française de Géotechnique, vol. 80, 1997, p. 17-34.

Peila D. Oreste P. P. Pelizza S. Poma A. "Study of the influence of sub-horizontal fiber-glass pipes on the stability of a tunnel face, $x$ Proc. Conf. North American Tunneling'96, Washington, Ed. Balkema, Rotterdam, 1996.

Spang K., Egger P. - " Action of fully-grouted bolts in jointed rock and factors of influence. is Rock Mechanics and Rock Engineering, vol. 23, 1990, p. 201-229.

Stille H. Holmberg M. Nord G. - a Support of weak rock with grouted bolts and shotcrete, $D$ Int. J. Rock Mech. Mining Sciences \& Geomech, vol. 26. 1989, p. 99-113

Subrin D, - «tude du comportement du front de taille d'un tunnel renforcé par des inclusions en fibre de verre, par voie analytique. ^ Mémoíre de DEA, École nationale des travaux publics de l'État, Lyon, 1997, $129 \mathrm{p}$.

Wong $\mathrm{H}$, Doanh I, - « EXTRUSION Computer program and accompanying user manual. „ École nationale des tra. vaux publics de l'État, Lyon, 1997.

Wong H., Jassionnesse C. - "Stabilité du front d'un tunnel renforcé par du boulonnage en fibre de verre, modèle analytique, » Proc. Symp. GEO, Aussois, France, 1996

Wong $\mathrm{H}$., Larue E. - a Modelling of bolting support in tunnels taking account of non-simultaneous yielding of bolts and ground. „x The Geotechnics of Hard Soils - Soft Rocks, Proc. IInd Int. Conf., Napoli 12-14 oct. 1998 , p. $1027-1038$

Wong H., Subrin D., Dias D. - a Extrusion movements of a tunnel head reinforced by finite length bolts - a closed form solution using homogenisation approach, s Int. J. of Num. \& Anal. Meth. in Geomech., 1999. 20 p.

Wong H., Subrin D., Dubois P. - a Mouve. ments en avant du front de taille d'un tunnel renforcé par boulonnage: influence du comportement du sol. ע Proc. Symp. GEO, Aussois, France, 24 28 nov. 1997.

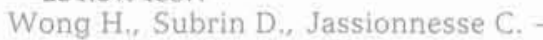
«Comportement du front d'un tunnel renforcé par du boulonnage en fibre de verre, modèle analytique, » Géomatériaux-Environnement-Ouvrages, vol. ' Ouvrages, Géomatériaux et Interactions, Ed. Hermès, France, 1998, p. 133-147.

Wong $H_{\text {., }}$ Trompille $\mathrm{V}_{.}$, Subrin D. Guilloux A, $-\alpha$ Tunnel face reinforced by longitudinal bolts : analytical model and in situ data. $n$ Proc. Int. Symp. on Geotechnical Aspects of Underground Construction in Soft Ground, Tokyo. 1999. 